\title{
AN ANALYSIS OF METAL FORMING PROCESSES USING LARGE DEFORMATION ELASTIC-PLASTIC FORMULATIONS*
}

\author{
Jung-Ho CHENG and Noboru KIKUCHI \\ Department of Mechanical Engineering and Applied Mechanics, The University of Michigan, Ann Arbor, \\ MI 48109, U.S.A.
}

Received 6 June 1984

\begin{abstract}
A concise survey of the literature related to the large deformation elasto-plasticity problems including unilateral contact and friction is presented together with an extension of the friction law for large deformation analysis.

Starting from the principle of virtual work, the so-called total Lagrangian and updated Lagrangian formulations are derived based on some fundamental assumptions in linearizing the nonlinear equations. By introducing the Zaremba-Jaumann (co-rotational) increment to the Cauchy stress tensor, the classical Prandtl-Reuss equations are generalized for describing the elastic-plastic material behavior. To allow a proper consideration of the contact conditions in the incremental analysis, a general friction law with an associated isotropic Coulomb sliding rule is obtained by the similarity between dry friction and plasticity. Finite element discretizations and approximations are applied to the resulting formulation of the updated Lagrangian approach.

Four example problems are solved to test the formulations developed in this paper. The emphasis is made toward the numerical accuracy of the finite element solutions.
\end{abstract}

\section{Introduction}

After two decades of development, finite element methods have been successfully applied to the analysis of many large deformation elasto-plastic problems. Since the establishment of finite element methods to solve linear problems, an extension to nonlinear elasto-plastic analysis was started by Argyris [1], Pope [2], Marcal and King [3], and Yamada et al. [4-5]. Stiffness matrices based on the incremental stress-strain relation were derived for small strain problems. Some techniques to control the transition region at the elastic-plastic interface were introduced. Another approach using an 'initial stress' computational process was proposed by Zienkiewicz et al. [6].

Extensive studies on the finite element analysis of finite strain problems were pursued by Turner et al. [7], Gallagher et al. [8], Kapur and Hartz [9], Martin [10-11], Oden [12-14], Besseling [15], Wissmann [16], Mallet and Marcal [17], Murray and Wilson [18], Marcal [19], Kawai [20], Hartz and Nathan [21], and Oden and Key [22]. It was not until Oden [23-24] that complete incremental forms were obtained from nonlinear continuum mechanics in which variations of loading and finite rotations of the element were properly considered. A survey of related works up to 1971 was given by Oden [25]. The first elasto-plastic formulation suitable

${ }^{*}$ This work was sponsored by the National Science Foundation under grant MEA-8109221. 
for large strain and large displacement was presented by Hibbitt, Marcal and Rice [26]. They used an incremental Lagrangian approach and pointed out that an additional 'initial stress' stiffness matrix, being dependent on current loads, was missed in ordinary small strain formulations. Later, McMeeking and Rice [27] derived an Eulerian type of finite element formulation following the principle of virtual work given by Hill [28]. The utilization of the updated finite clement mesh as the reference configuration made this approach very appealing to investigators in the area of large deformation analysis, especially with some path-dependent materials. Alternative formulations and implementations are due to Needleman [29], Larsen and Popov [30], Key [31], Osias and Swedlow [32], Bathe, Ramm and Wilson [33], Kleiber [34], Wunderlich [35], Argyris et al. [36-37], Cescotto et al. [38], Atluri [39-40], Gadala et al. [41], and Mattiasson [42].

The number of applications to metal forming problems and structural analysis is tremendous. Needleman [29], McMeeking and Rice [27], and Chen [43] have extensively studied the simulation of necking phenomena in a simple tension test. Stress distributions in a steady-state extrusion process were obtained by Lee, Mallett and Yang [44], and Lee, Mallett and McMeeking [45]. Upsetting problems were investigated by Yamada, Wifi and Hirakawa [46], Nagtegaal and De Jong [47], and Boer et al. [48]. A summary and comparison of different computer codes applied to an axisymmetric upsetting problem were presented by Kudo and Matsubara [49]. Intensive analyses of sheet metal forming were treated by Gotoh [50], Wang and Budiansky [51], Oh and Kobayashi [52], Wifi [53-54], and others, A variety of forming processes has been successfully solved by Key, Krieg and Bathe [55], Argyris and Doltsinis [56-58], Argyris et al. [59], Taylor [60], and Kikuchi and Cheng [61]. Among these, the aspect of thermomechanical coupling was taken into account in [58], and contact conditions with friction were included in the last two papers. In structural mechanics, nonlinear elasto-plastic large deformation analyses have also attracted a considerable amount of research efforts from many authors such as Yaghmai and Popov [62], Dupuis et al. [63], Heifitz and Constantino [64], Bathe et al. [33, 65-66], and Argyris et al. [67], to name a few.

In addition to those works cited above, two major alternatives were introduced for the analysis of metal forming processes. First, as being advocated by Kobayashi and his collaborators [68-70], the rigid-plastic formulations in which the elastic deformations are assumed to be negligible compared to the large plastic strains, have gained a wide range of applications. The method fails to predict the stress history whenever elastic loading or unloading is encountered. The second approach owing to Zienkiewicz et al. [71-75] (the inclusion of elastic strains was presented by Dawson and Thompson [76]), which is based on a viscoplastic formulation and thus enables itself to deal with rate-dependent materials, has also acquired relatively successful small strain analysis of different processes.

Solutions of nonlinear elasto-plastic problems strongly depend on the use of finite element discretization and incremental strategy. Several different methods have been discussed by Ricc and Tracey [77], Krieg and Krieg [78], Schreyer et al. [79], Crisfield [80], Tracey and Freese [81], and Nyssen [82] for integration of the constitutive equations. The overconstrained problems of the finite element model due to the incompressibility condition of the plastic deformation have been carefully examined by many authors. Hermann [83] developed a special formulation for incompressible or nearly incompressible materials. Nagtegaal, Parks and Rice [84] observed that the 'locking phenomenon' can be overcome by special arrangements of elements so as to eliminate one constraint. Another approach called the selective 
reduced integration method was suggested by Zienkiewicz, Taylor and Too [85], Pawsey and Clough [86], and Naylor [87] to properly choose less order integration points so that there exists an adequate total number of degrees of freedom. Currently used methods and difficulties for finite element analysis of large strain plasticity problems were reviewed by Bathe et al. [88], and Nagtegaal and Veldpaus [89].

Another very important characteristic in most forming processes is the existence of contact conditions with friction. The variational theory of contact mechanics was initiated by Signorini [90] in 1959 and the problem was solved by Fichera [91] in 1963. Kalker [92] in a review paper provided a summary of the theory of contact mechanics prior to 1974. Finite element analyses to this subject formulated by variational inequalities were studied by Fredriksson, Rydholm and Sjoblom [93], Hlavacek and Loisek [94], Kikuchi and Oden [95], Kikuchi and Song [96], Kikuchi and Skalski [97], and Oden and Kikuchi [98]. Various algorithms and numerical implementations to solve elastic contact problems were presented by Chan and Tuba [99], Tsuta and Yamaji [100], Ohte [101], Francavilla and Zienkiewicz [102], Okabe and Kikuchi [103], Sachdeva and Ramakrishnan [104], Zeid and Padovan [105], Mazurkiewicz and Ostachowicz [106], and Gurra and Browing [107]. Either the Lagrange multiplier method or the penalty method was used to resolve the constrained boundary conditions. Schäfer [108], and Yamada et al. [109] proposed a technique using the concept of bond elements. Applications to the dynamics of impact problems were considered by Hughes et al. [110], Talaslidis and Panagiotopoulos [111], and Osmont [112]. The use of the incremental theory in obtaining the friction law was adopted by Seguchi et al. [113], Fredriksson [114], Peterson [115], Michalowski and Mroz [116], and Okamoto and Nakazawa [117]. A concise survey including both mathematical development and practical considerations was given in the treatise by Kikuchi and Oden [95].

In the present paper, starting from the principle of virtual work, the boundary value problems of arbitrary amount of deformation are formulated in the deformed configuration. By properly choosing the reference state, transformation and linearization of the nonlinear equations result in two well-known incremental forms; the total Lagrangian formulation and the updated Lagrangian formulation. To describe the material behavior, the Prandtl-Reuss equations based on $\boldsymbol{J}_{2}$ flow theory of von-Mises type are assumed and are generalized for large deformation analysis. As the nature of nonlinear problems, many models exist in the literature of the theory of plasticity. Among others, $J_{2}$ deformation theory originated by Hencky [118] and reassessed by Budiansky [119] has been frequently argued to not being suitable to describe the complete plastic behavior of a metal except for nearly proportional stress increments. A modified version termed the $J_{2}$ corner theory was given by Christoffersen and Hutchinson [120] to circumvent some of the difficulties associated with the bifurcation-related phenomena in the plastic range. Many other constitutive equations for elastic-plastic materials at finite strain have been proposed by Freund [121], Tanaka [122], Hahn [123], Key, Biffle and Krieg [124], Shiratori, Ikegami and Yoshida [125], Axelsson and Samuelsson [126], Dashner [127], Hashiguchi [128], Mandel [129], Nemat-Nasser [130], Sidoroff [131], Tokuora [132], Key and Krieg [133], Lee, Mallett and Wertheimer [134], Palgen and Drucker [135], and Atluri [136]. Mixed hardening of isotropic with kinematic has been studied in many of these works. Recently, a summary of some of the major theories in finite plasticity of crystalline solids and geomaterials was presented by Nemat-Nasser [137] from both microscopic and macroscopic phenomenological points of view. However, due to Hill's treatise [138] and the paper by 
Hibbitt et al. [26], the generalized Prandtl-Reuss equations still remain most popular in applications.

To allow for the effect of finite rotation and to satisfy the requirement of objectivity in a material constitutive law, the Zaremba-Jaumann increment (or rate) of the Cauchy stress tensor is chosen to be a function of the strain-increment (rate) tensor. While there have been many more stress rates proposed by, for example, Oldroyd [139], Cotter and Rivlin [140], and Truesdell [141], the question of which measure is superior to others is still a controversial topic. The definition of Zaremba-Jaumann's (or co-rotational) rate seems to be more preferable than many available because, as shown in Prager [142], the vanishing of the ZarembaJaumann's stress rate does (but others do not) imply the stationary invariants of the stress tensor. Johnson and Bammann [143], Lee and Wertheimer [144], Atluri [136], and Dafalias [145] have discussed the features of some different stress rate measures.

Following the concepts in [95] for dealing with normal contact, the formulation of the principle of virtual work is completed by introducing a friction law similar to the ones deduced by Seguchi et al. [113] and Fredriksson [114], in which the analogy with the plasticity theory has been used. However, in these studies a basic assumption is made, namely, that the normal reaction is constant during the deformation process, which is generally not valid in the incremental analysis of metal forming processes. Recognizing this fact, an incremental friction law including the contribution of normal contact pressure is derived and can be readily incorporated into the computer code of incremental type. One interesting observation of the friction law obtained in this paper is that it can be directly related to the experimental results on the coefficient of friction which show the 'hardening' effect similar to the plastic behavior of materials.

Some bench mark problems are chosen to examine the numerical solutions obtained from the computer code 'EPAFRIC' (Elastic-Plastic Analysis of FRIction Contact problems) developed by the authors. Several delicate problems such as the importance in selecting the finite element discretization and the size of increments are carefully investigated and discussed.

\section{Field equations}

For a deformed body in equilibrium, once the constitutive law (stress-strain relation), is chosen, the stress field can be determined by solving the following governing equations:

$$
\sigma_{j i, j}+\rho b_{i}=0 \quad \text { in } \Omega,
$$

satisfying the boundary conditions

and

$$
u_{i}-g_{i} \text { on } \Gamma_{\mathrm{D}}
$$

$$
\sigma_{i i} n_{j}=t_{i} \quad \text { on } \Gamma_{\mathrm{F}} .
$$

where $\sigma\left(=\sigma_{j i} \boldsymbol{e}_{j} \boldsymbol{e}_{i}\right)$ is the true (Cauchy) stress tensor defined in the deformed configuration $\Omega$ which is depicted in a fixed rectangular Cartesian coordinate system $\boldsymbol{x}$ with the origin $o$ and 


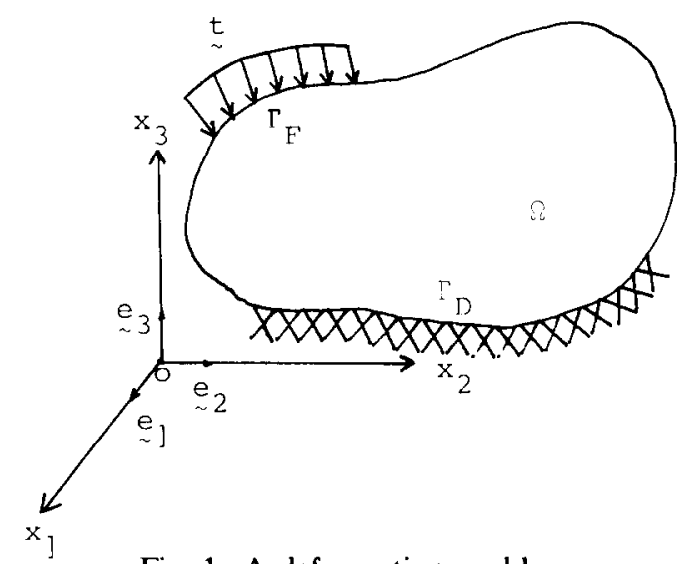

Fig. 1. A deformation problem.

base vectors $\boldsymbol{e}_{i}$ (Fig. 1), the comma notation represents the partial derivatives with respect to the coordinates $x_{i}$, i.e., $\sigma_{j i, j}=\partial \sigma_{j i} / \partial x_{j}, \rho$ is the mass density function, $\boldsymbol{b}\left(=b_{i} \boldsymbol{e}_{i}\right)$ is the body force vector, $\boldsymbol{u}\left(=u_{i} e_{i}\right)$ is the displacement vector which is specified by a given vector function $g$ $\left(=g_{i} e_{i}\right)$ on the portion $\Gamma_{\mathrm{D}}$ and $t\left(=t_{i} \boldsymbol{e}_{i}\right)$ is the traction vector function prescribed on the surface $\Gamma_{\mathrm{F}}$ with a unit normal vector $\tilde{\boldsymbol{n}}\left(=\tilde{n}_{i} e_{i}\right)$.

Throughout this paper, all vector and tensor components are Cartesian. The summation convention is used. All dummy indices which appear exactly twice in a given term will assume summation over the values 1 to 3 unless otherwise stated.

\section{Weak form (the principle of virtual work)}

Another way to state the boundary value problem described above is to apply the principle of virtual work to the local form (1). Let $\bar{u}\left(=\bar{u}_{i} e_{i}\right)$ to an arbitrary virtual (variation of) displacement vector such that $\bar{u}_{i}=0$ on $\Gamma_{\mathrm{D}}$. Multiplying $\bar{u}_{i}$ to both sides of (1), taking the integration over the domain $\Omega$, and applying the divergence theorem, we have

$$
\int_{\Omega} \sigma_{i j} \bar{u}_{i, j} \mathrm{~d} \Omega=\int_{\Omega} \rho b_{i} \bar{u}_{i} \mathrm{~d} \Omega+\int_{\Gamma_{\mathrm{F}}} t_{i} \bar{u}_{i} \mathrm{~d} \Gamma \quad \forall \bar{u}_{i} \text { such that } \bar{u}_{i}=0 \text { on } \Gamma_{\mathrm{D}} .
$$

Here we have used the boundary conditions (2) and (3) on $\Gamma$ which is assumed at this moment to consist of two disjointed portions $\Gamma_{\mathrm{D}}$ and $\Gamma_{\mathrm{F}}$. Later the boundary conditions of unilateral contact with friction will be included and discussed in detail.

Direct applications of (4) are limited to linear problems where the deformations are small and the materials respond linearly during the deformation. Equation (4) is formulated in the deformed configuration $\Omega$. If the deformation of the body is infinitesimally small, this expression may be used without difficulty by taking $\Omega$ to be essentially the same as the initial (stress free) configuration $\Omega^{0}$. However, when the deformation is finite, $\Omega$ is in general considerably different from $\Omega^{0}$ and is one of the unknowns to be determined. Therefore, it will be more convenient if (4) can be transformed and expressed in the reference configuration $\Omega_{\mathrm{R}}$ which may be identified with the initial one $\Omega^{0}$. 
Writing the principle of virtual work in the reference configuration is also essential in the sense that the domain can be held fixed when later we have to take linear increments to nonlinear equations.

\section{The total Lagrangian formulation}

Consider a body occupying a region $\Omega^{0}$ with boundary $\Gamma^{0}$ initially, which corresponds to a region $\Omega$ with boundary $\Gamma$ in the deformed state (Fig. 2). The position of any material point in the bodies in the reference and deformed configurations can be traced by its radius vectors $\boldsymbol{X}$ $\left(=X_{I} e_{I}\right)$ and $x\left(=x_{i} e_{i}\right)$, respectively. Capitalized indices indicate measures with respect to the reference state. The difference between $\boldsymbol{x}$ and $\boldsymbol{X}$ is defined by the displacement vector $\boldsymbol{u}$ with the components

$$
u_{i}=x_{i}-X_{i}
$$

During the deformation, the mass is conserved, i.e.,

$$
\int_{\Omega} \rho \mathrm{d} V=\int_{\Omega^{0}} \rho^{0} \mathrm{~d} V^{0},
$$

where $\mathrm{d} V$ and $\mathrm{d} V^{0}$ are infinitesimal volume elements.

Realizing that any quantity of a material point can be represented by a function of either $\boldsymbol{x}$ in the deformed configuration or $\boldsymbol{X}$ in the initial configuration provided that the relation between $\boldsymbol{x}$ and $\boldsymbol{X}$ is uniquely defined, we can use (6) to transform (4) from $\Omega(\Gamma)$ to $\Omega^{0}\left(\Gamma^{0}\right)$ as

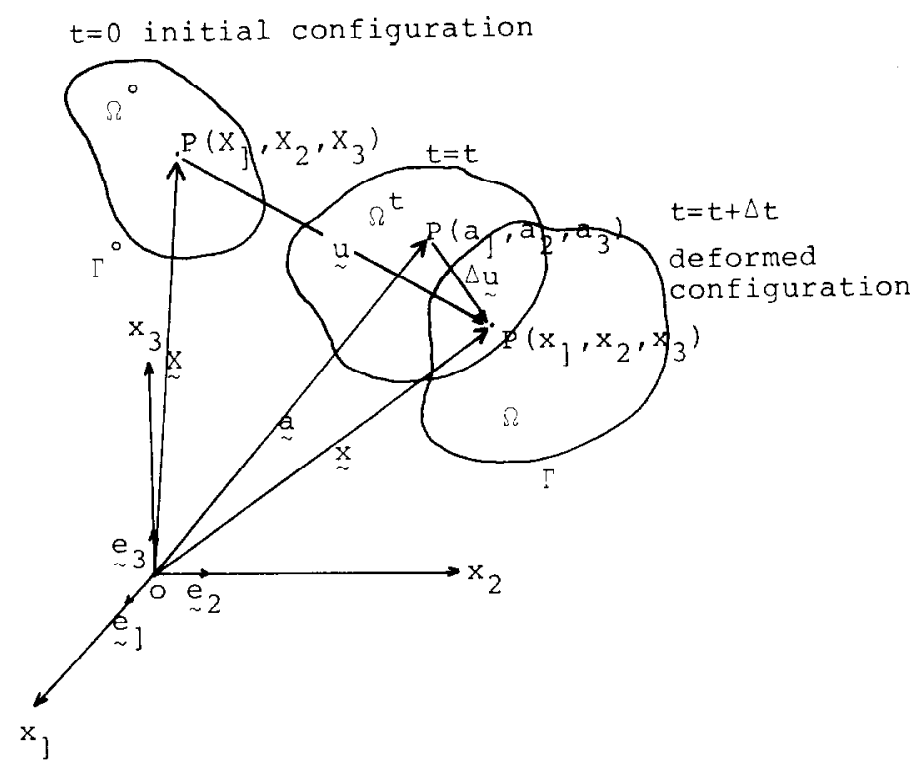

Fig. 2. Reference configurations for the large deformation analysis. 
follows:

$$
\int_{\Omega^{0}} T_{f i} \bar{u}_{i, J} \mathrm{~d} \Omega^{0}=\int_{\Omega^{0}} \rho^{0} b_{i} \bar{u}_{i} \mathrm{~d} \Omega^{0}+\int_{\Gamma^{0}} t_{i}^{0} \bar{u}_{i} \mathrm{~d} \Gamma^{0} .
$$

where $\boldsymbol{T}\left(=T_{J i} e_{s} e_{i}\right)$ is the first Piola-Kirchhoff (Lagrange, nominal) stress tensor related to the Cauchy stress tensor $\sigma_{j i}$ by

$$
T_{J i}=J X_{J, j} \sigma_{j i}
$$

$t^{0}\left(=t_{i}^{0} e_{i}\right)$ is the traction vector given on the undeformed boundary surface $\Gamma_{\mathrm{F}}, J\left(=\rho^{0} / \rho\right)$ in (8) is the determinant of the Jacobian matrix $\left[\partial x_{i} / \partial X_{j}\right]$. It is noted that $u_{i,}$ means the partial derivative of the $i$ th component of $\boldsymbol{u}$ with respect to the (material) coordinate $X_{J}$ defined in the reference configuration $\Omega^{0}$. Indeed, for an arbitrary function $f$ associated to mass (or a particle) of the body, the relation

$$
\int_{\Omega} \rho f(x) \mathrm{d} \Omega=\int_{\Omega^{0}} \rho^{0} \hat{f}(X) \mathrm{d} \Omega^{0}, \quad \hat{f}(\boldsymbol{X})=f(x(X))
$$

represents the transformation between the deformed and reference configurations. Thus the internal virtual work can be written as

$$
\int_{\Omega} \sigma_{j i} \bar{u}_{i, j} \mathrm{~d} \Omega=\int_{\Omega} \rho^{-1} \rho \sigma_{j i} X_{J, j} \bar{u}_{i, J} \mathrm{~d} \Omega=\int_{\Omega^{\mathrm{0}}} \rho^{0} \rho^{-1} \sigma_{j i} a_{J, j} \bar{u}_{i, i} \mathrm{~d} \Omega^{\mathrm{o}} .
$$

It follows from $J \rho=\rho^{0}$ that the virtual work principle (4) in the deformed configuration is transformed into (7) in the reference configuration.

It is noted, from the balance of the moment of momentum that the Cauchy stress tensor is symmetric, i.e., $\sigma_{j i}=\sigma_{i j}$. However, the relation (8) implies that the Lagrange stress tensor is not symmetric. In order to avoid managing the unsymmetric tensor, let us define another stress measure as

or

$$
S_{J I}=X_{I, i} T_{J i},
$$

$$
S_{J I}=J X_{l, i} X_{J, j} \sigma_{j i} .
$$

where $S=S_{J I} e_{J} e_{I}$ is the second Piola-Kirchhoff (or Kirchhoff) stress tensor which has the desired symmetry property. Then (7) can also be expressed as

$$
\int_{\Omega^{0}} S_{J J} x_{i, J} \bar{u}_{i, J} \mathrm{~d} \Omega^{0}=\int_{\Omega^{0}} \rho^{0} b_{i} \bar{u}_{i} \mathrm{~d} \Omega^{0}+\int_{\Gamma_{\mathrm{F}}^{0}} t_{i}^{0} \bar{u}_{i} \mathrm{~d} \Gamma^{0},
$$

which is called the Lagrangian formulation [26], or the total Lagrangian formulation [33] to the boundary value problem (1)-(3), with $\Omega^{0}$ being identified as the initial configuration of the body. If we recall that the variation of Green's strain tensor 


$$
e_{I J}=\frac{1}{2}\left(x_{i, I} x_{i, J}-\delta_{I J}\right)
$$

is given by

$$
\bar{e}_{I J}=\frac{1}{2}\left(x_{i, I} \bar{u}_{i, J}+\bar{u}_{i, I} x_{i, J}\right),
$$

then symmetry of the Kirchhoff stress tensor yields

$$
S_{J I} x_{i, I} \bar{u}_{i, J}=S_{J I} \bar{e}_{I J}
$$

and the meaning of the left-hand side of (11) becomes clear.

\section{Incremental forms}

Linearity of the governing equations is always a great advantage in solving boundary value problems. Unfortunately, for large deformations and general material properties, the equations are highly nonlinear. Therefore, in many applications it is convenient to derive linearized forms of these equations under the assumption that the entire nonlinear response of the body is replaced by a sequence of piecewise linear increments.

Suppose that at time $t$ a deformed body occupies a configuration $\Omega^{t}$. At a later instant $t+\Delta t$, the body is further deformed to coincide with $\Omega$ which is sufficiently close to $\Omega^{t}$ (Fig. 2). The stresses in the body and coordinates of each point change from $S_{I I}$ to $S_{J I}+\Delta S_{J I}$ and from $x_{i}$ to $x_{i}+\Delta u_{i}$, respectively, due to the increments of $\Delta b_{i}$ and $\Delta t_{i}^{0}$. Thus, for each successive increment, (11) takes the form, by neglecting the higher-order terms $\Delta S_{J I} \Delta u_{i, I}$ and $\Delta S_{J I} \Delta u_{i, J}$, of

where

$$
\int_{\Omega^{0}}\left(\Delta S_{J I} x_{i, I} \bar{u}_{i, J}+S_{J I} \Delta u_{i, J} \bar{u}_{i, J}\right) \mathrm{d} \Omega^{0}=R+\int_{\Omega^{0}} \rho^{0} \Delta b_{i} \bar{u}_{i} \mathrm{~d} \Omega^{0}+\int_{\Gamma_{\mathrm{F}}^{0}} \Delta t_{i}^{0} \bar{u}_{i} \mathrm{~d} \Gamma^{0},
$$

$$
R=-\int_{\Omega^{0}} S_{J I} x_{i, I} \bar{u}_{i, J} \mathrm{~d} \Omega^{0}+\int_{\Omega^{0}} \rho^{0} b_{i} \bar{u}_{i} \mathrm{~d} \Omega^{0}+\int_{\Gamma_{\mathrm{F}}^{0}} t_{i}^{0} \bar{u}_{i} \mathrm{~d} \Gamma^{0}
$$

is the remainder (load corrector) emerging from the unbalance of the resultant force. For a simple incremental solution, $R$ is taken to be zero. The improvement by this correction has been investigated by Yamada et al. [46] and Wifi [54].

Discussions on how to obtain proper forms of $\Delta b_{i}$ and $\Delta t_{i}$ can be found in Hibbitt et al. [26]. Here we shall be concerned with the case of pressure loading which is important in many practical applications, especially in the analysis of hydrostatic bulging of sheet metals. If the pressure acting on the surface with the unit outward normal $\tilde{\boldsymbol{n}}$ is $p(p>0)$, then the second term of (4) becomes

$$
\int_{\Gamma_{\mathrm{F}}} t_{i} \bar{u}_{i} \mathrm{~d} \Gamma=\int_{\Gamma_{\mathrm{F}}}-p \tilde{n}_{i} \bar{u}_{i} \mathrm{~d} \Gamma
$$

From vector analysis (see Spencer [146, p. 124]), we have 


$$
\mathrm{d} s \tilde{n}_{i}=J X_{I, i} \mathrm{~d} s^{0} \tilde{n}_{I}^{0},
$$

where $\mathrm{d} s(=\mathrm{d} \Gamma)$ and $\mathrm{d} s^{0}\left(=\mathrm{d} \Gamma^{0}\right)$ are differential elements of $\Gamma$ and $\Gamma^{0}$, respectively.

Substituting (18) into (17) and comparing with (11), we obtain

$$
t_{i}^{0}=-p J X_{I, i} \tilde{n}_{I}^{0} .
$$

The incremental form can be written as

$$
\Delta t_{i}^{0}=-\Delta p J X_{I, i} \tilde{n}_{I}^{0}-p \Delta\left(J X_{I, i}\right) \tilde{n}_{I}^{0},
$$

in which the second term is due to the change in geometry with the load held fixed. It is noted that the second term generates additional terms to the stiffness matrix in finite element analysis since $\Delta J$ and $\Delta X_{I, i}$ are related to $\Delta u_{i, I}$.

\section{The updated Lagrangian formulation}

If instead of dealing with (7), we take the configuration $\Omega^{t}$ at time $t$ as the reference state. All static and kinematic variables are referred to this configuration in which $S_{I I} \simeq T_{J i} \simeq \sigma_{j i}$ is assumed. Fig. 2 illustrates relations among the initial configuration $\Omega^{0}$, the reference configuration $\Omega^{t}$, and the current (deformed) configuration $\Omega$, where $\boldsymbol{X}, \boldsymbol{a}$ and $\boldsymbol{x}$ are selected to describe the position of any material point in these configurations. For an increment $\Delta t$ small enough so that $J \approx 1$ instantaneously, (7) can be written in an incremental form as

$$
\int_{\Omega^{t}} \Delta T_{j i} \bar{u}_{i, J} \mathrm{~d} \Omega^{t}=\int_{\Omega^{\prime}} \rho \Delta b_{i} \bar{u}_{i} \mathrm{~d} \Omega^{t}+\int_{\Gamma_{F}^{t}} \Delta t_{i} \bar{u}_{i} \mathrm{~d} \Gamma^{t},
$$

which is termed the Eulerian formulation by McMeeking and Rice [27] although in their work the rate form was used, which is equivalent to the present form if the Euler's scheme is adopted to integrate it over the time domain. Bathe et al. [33], however, obtained another incremental form based on (11) in terms of $\Delta S_{J I}$ and called it the updated Lagrangian formulation due to the choice of the updated reference state.

The relations between $\Delta T_{J_{i}}$ and $\Delta \sigma_{j i}$ can be obtained by taking increment on (8),

$$
\Delta T_{J i}=\Delta \sigma_{j i} \delta_{J j}+\sigma_{J i} \Delta u_{k, k}-\sigma_{j i} \Delta u_{J, j},
$$

in which some basic assumptions have been made: $J \approx 1, \Delta J \approx \Delta u_{k, k}, a_{J_{, j}} \approx \delta_{j,}, \Delta a_{J, j} \approx-\Delta u_{j, j}$.

Using (22) or the similar approach in the previous section, the increment of the traction vector for a type of pressure loading can be expressed as

$$
\Delta t_{i}=-\Delta p \tilde{n}_{i}-p \Delta u_{k, k} \tilde{n}_{i}+p \Delta u_{i, i} \tilde{n}_{I},
$$

under the assumption that the unit normal $\tilde{n}$ does not change its direction during the increment, where $p$ is the pressure in the reference state $\Omega^{t}$.

In the following discussions, we shall use (21) exclusively in the analysis of large elasto- 
plastic deformation problems. The reasons of this choice are that the equations of the updated Lagrangian formulation are obviously simpler than those of the total Lagrangian formulation. and that for history-dependent elasto-plastic materials the constitutive relations are readily incorporated in the updated Lagrangian formulation since these equations are functions of variables referred to the current state, although these can be transformed into the ones in the initial configuration.

Theoretically these is no difference between the total Lagrangian and updated Lagrangian formulations. Comparisons of these two approaches have been presented by Cescotto et al. [38], Atluri [39] and Gadala et al. [41] from the aspect of formulations, and also by Bathe et al. $[33,65]$ and Mattiasson [42] from the view-point of numerical effectiveness.

\section{Constitutive relations}

By introducing the Zaremba-Jaumann increment $(\nabla)$ to the Cauchy stress tensor $\sigma_{i j}$ such that

$$
\sigma_{i j}^{\nabla}=\Delta \sigma_{i j}-\sigma_{i k} \Omega_{k j}-\sigma_{j k} \Omega_{k i},
$$

where $\Omega_{i j}=\frac{1}{2}\left(\Delta u_{j, i}-\Delta u_{i, j}\right)$ is the spin tensor, the constitutive equation of an isotropic material (excluding the thermal effect) can be written as

$$
\sigma_{i j}^{\Gamma}=\Delta \varepsilon_{k k}^{e} \delta_{i j}+2 G \Delta \varepsilon_{i j}^{e},
$$

where $\lambda$ and $G$ are 1st and 2nd Lamé constants and $\Delta \varepsilon_{i j}^{e}$ are the elastic components of the strain-increment tensor $\Delta \varepsilon_{i j}$ defined by

$$
\Delta \varepsilon_{i j}=\frac{1}{2}\left(\Delta u_{i, j}+\Delta u_{j, i}\right) .
$$

Basic postulates [137] in the plasticity theory suggested by Levy and von Mises, later extended by Prandtl and Reuss are: (a) There exists a yield function

$$
f\left(\sigma_{i}, Y\right)=\sqrt{3 J_{2}}-Y,
$$

such that $f<0$ implies elastic loading and the material is said to be yielded when $f=0$, where $J_{2}\left(=\frac{1}{2} s_{i j} s_{i j}\right)$ is the second invariant of the deviatoric stress tensor $s_{i j}\left(=\sigma_{i j}-\frac{1}{3} \delta_{i j} \sigma_{k k}\right)$, and $Y$ is the yield strength depending on the strain history. (b) An associated isotropic-hardening (thus the Bauschinger effect is not considered) flow rule

$$
\Delta \varepsilon_{i j}^{\mathrm{p}}=\Lambda \partial f / \partial \sigma_{i j}
$$

is applied to describe the postyield behavior, where $A$ is a positive scalar function. (c) The decomposition of $\Delta \varepsilon_{i j}$ into $\Delta \varepsilon_{i j}^{e}$ and $\Delta \varepsilon_{i j}^{\text {p }}$, i.e.,

$$
\Delta \varepsilon_{i j}=\Delta \varepsilon_{i j}^{\mathrm{c}}+\Delta \varepsilon_{i j}^{\mathrm{p}},
$$


is assumed to be held throughout the entire deformation process. The validity of this assumption in a finite elasto-plasticity theory has been thoroughly discussed by Nemat-Nasser $[130,147]$. Another theory which is in contrast with this model was proposed by Lee [148-149] based on the decomposition of the total deformation gradient to an elastic and plastic parts. Arguments on this controversy can be directed to Green and Naghdi [150], Casey and Naghdi [151], and Lee and McMeeking [152] as well.

Using (25), (27), (28) and (29), the Prandtl-Reuss stress-strain relations for materials obeying von-Mises yield criterion can be deduced [4] as

where

$$
\sigma_{i j}^{\nabla}-D_{i j k l} \Delta \varepsilon_{k l},
$$

$$
D_{i j k l}=(E \nu /(1+\nu)(1-2 \nu)) \delta_{i j} \delta_{k l}+G\left(\delta_{i k} \delta_{j l}+\delta_{i l} \delta_{j k}\right)-\alpha 3 G\left(s_{i j} s_{k l}\right) /\left(\bar{\sigma}^{2}\left(1+H^{\prime} / 3 G\right)\right)
$$

is the material constant tensor, $E, G, \nu$ are the Young's modulus, the shear modulus and the Poisson's ratio, respectively, $\bar{\sigma}\left(=\sqrt{3 J_{2}}\right)$ is the effective (or equivalent) stress, $H^{\prime}=$ $H_{0}^{\prime} /\left(1-H_{0}^{\prime} / E\right), H_{0}^{\prime}$ is the tangent of the true stress-logarithmic strain curve, and $\alpha=0$ if $f<0$ (elastic loading) or $f=0$ but $s_{i j} \Delta \varepsilon_{i j}<0$ (unloading); otherwise $\alpha=1$ if $f=0$ and $s_{i j} \Delta \varepsilon_{i j} \geqslant 0$ (plastic loading). Loading and unloading criteria follow directly from whether $f^{\nabla}=0$ or $f^{\nabla}<0$ for next increment (see discussions by Fung [153, p. 140] or Malvern [154, p. 368]).

Since the term $\sigma_{j i} \Delta u_{k, k}$ in (22) yields unsymmetric stiffness matrices in the finite element approximation to the principle of virtual work (21), we shall replace the constitutive equation (30) by

$$
\sigma_{i j}^{\nabla}+\sigma_{i j} \Delta u_{n, n}=\hat{D}_{i j k l} \Delta \varepsilon_{k l}
$$

which leads to a symmetric stiffness matrix, $\hat{D}_{i j k l}$ is the same as (31) except that $H^{\prime}$ has to be changed into

$$
\hat{H}^{\prime}=H_{0}^{\prime} /\left(1-H_{0}^{\prime} / E-(1-2 \nu) \bar{\sigma} / E\right) .
$$

The difference between $\hat{D}_{i j k l}$ and $D_{i j k l}$ is $(1-2 \nu) /(2 G(1+\nu))$ which is within the order of elastic strain and is generally negligible in the finite deformation analysis. See discussions by Tomita [155].

\section{Unilateral contact conditions}

Without loss of generality let us consider a rigid body moving on a deformable body of which the surface can be represented by a parametric equation

$$
x_{3}=\phi\left(x_{1}, x_{2}\right) .
$$

If the rigid surface can also be described as

$$
x_{3}=\psi\left(\hat{x}_{1}, \hat{x}_{2}\right),
$$


parametrically where a $\hat{\prime}$ on the coordinates $x_{1}, x_{2}$ indicates the updated coordinates of the deformable surface taking account of the relative motions of the origins of $\phi$ and $\psi$ (Fig. 3). Then the kinematic contact condition,

$$
\phi\left(x_{1}, x_{2}\right)+v_{3}(x) \Delta t \leqslant \psi\left(x_{1}+\left(v_{1}(x)-v_{1}^{\mathrm{R}}\right) \Delta t, x_{2}+\left(v_{2}(x)-v_{2}^{\mathrm{R}}\right) \Delta t\right)+v_{3}^{\mathrm{R}} \Delta t,
$$

has to be satisfied for an arbitrary time increment $\Delta t$ on the possible contact surface $\Gamma_{\mathrm{c}}^{t}$, where $v\left(=v_{i} e_{i}\right)$ is the velocity vector of the point with the updated coordinates $x\left(x_{1}, x_{2}, \phi\left(x_{1}, x_{2}\right)\right)$ on the deformable surface, and $v^{\mathrm{R}}\left(=v_{i}^{\mathrm{R}} \boldsymbol{e}_{i}\right)$ is the prescribed velocity vector of the rigid body.

The equality of (36) can be used to solve for a proper $\Delta t$ so as to just bring a (or some) point(s) to be in contact with the rigid surface. If the increment $\Delta t$ is small enough, (36) can be linearized as

$$
n \cdot v \Delta t \leqslant n_{3}(\phi-\psi)+v^{\mathrm{R}} \cdot n \Delta t,
$$

where $n\left(=n_{i} \boldsymbol{e}_{i}\right)$ is the unit vector inward normal to the rigid surface defined by

$$
n=\left\{-e_{1}\left(\partial \psi / \partial x_{1}\right)-e_{2}\left(\partial \psi / \partial x_{2}\right)+e_{3}\right\} / \sqrt{\left(\partial \psi / \partial x_{1}\right)^{2}+\left(\partial \psi / \partial x_{2}\right)^{2}+1}
$$

After the point is in contact with the rigid surface, $\phi=\psi$ instantaneously; therefore, (34) becomes

$$
\Delta u_{n}-\Delta g_{n}=0 \text { on } \Gamma_{c}^{*},
$$

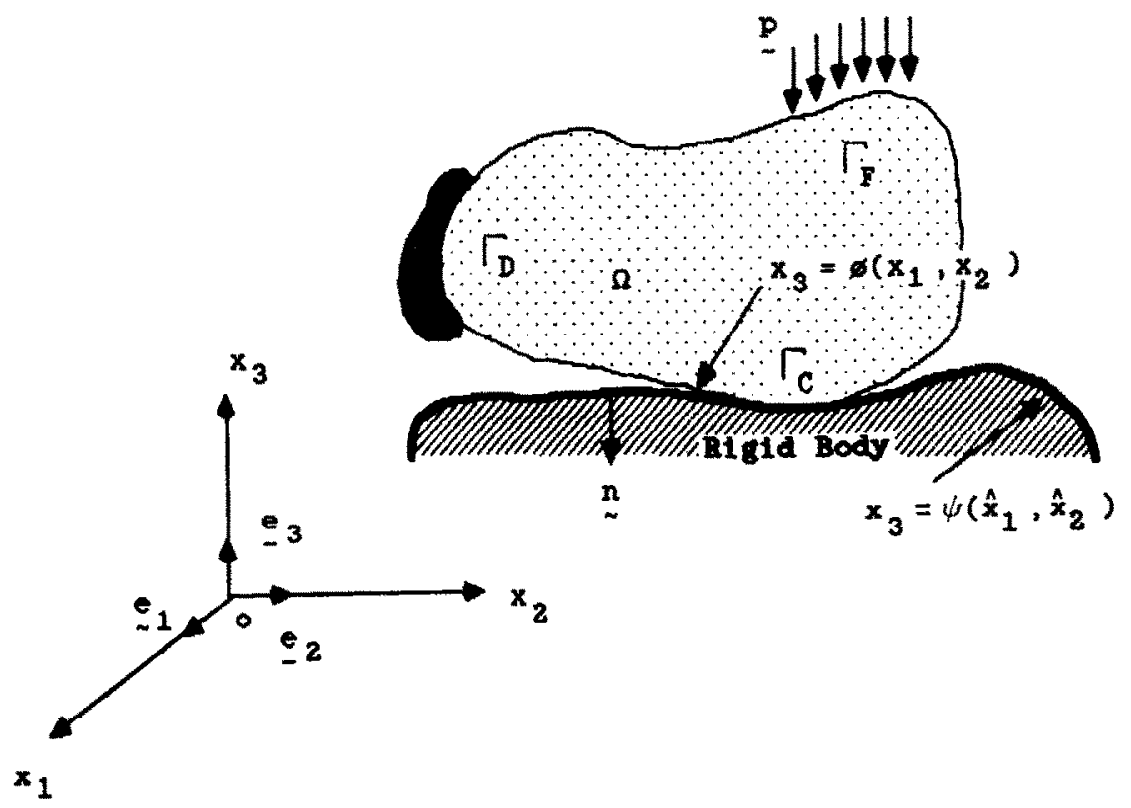

Fig. 3. A unilateral contact problem. 
where $\Delta u_{n}(=\Delta \boldsymbol{u} \cdot \boldsymbol{n})$ is the increment of the normal displacement constrained by a given motion $\Delta g_{n}$ on the in-contact surface $I_{c}^{*}$.

In order to resolve the constraint (39), we shall apply the exterior penalty method introduced by Courant, Friedrichs and Lewy [156], and extended by Zangwill [157]. As Courant [158] indicated, physically the constraint (39) can be viewed as the existence of a very stiff spring between the contact surfaces. Thus, if we define

$$
\Delta t_{n}=-k_{n}\left(\Delta u_{n}-\Delta g_{n}\right) \text { on } \Gamma_{\mathrm{c}}^{*}
$$

with a very large penalty parameter $k_{n}(>0$, spring constant), then (39) is replaced by a third type of boundary condition approximately. Furthermore, the increment of normal reaction $\Delta t_{n}$ $(<0)$ is obtained as a by-product from (37). Depending upon the loading condition a point which is already on $\Gamma_{\mathrm{c}}^{*}$ may tend to leave the rigid surface at certain deformation stage; in this case the sign of $\Delta t_{n}$ becomes positive. Nevertheless, the point will stay in contact as long as the resultant contact pressure $t_{n}$ updated by $\Delta t_{n}$ remains negative.

The proofs of the existence of the solution to the penalized problem and its convergence to the original problem (39) as $k_{n} \rightarrow+\infty$ have been given by Kikuchi and Song [96]. The extension of this idea to various unilateral contact problems can also be found in [95].

\section{An incremental friction law}

Friction forces arise whenever two contact surfaces are in relative motion. A relation among the increment of the tangential friction force, the displacement increment, and the increment of the contact pressure can be derived using the similarity of sticking-sliding phenomena and elastic-plastic behaviors as shown by Seguchi et al. [113] and Fredriksson [114]. If we assume that there exists a Coulomb isotropic slip function [114],

$$
f\left(t_{\mathrm{T}}, \mu_{\mathrm{F}}, t_{n}\right)=\sqrt{\left(t_{\mathrm{T} i} t_{\mathrm{Ti}}\right)}+\mu_{\mathrm{F}} t_{n},
$$

where $t_{\mathrm{T}}\left(=t_{\mathrm{T} i} e_{i}\right)$ and $t_{n}$ are the tangential and normal components of the traction vector $t$ $\left(=t_{\mathrm{T}}+t_{n} n\right)$, and $\mu_{\mathrm{F}}$ is the coefficient of friction. Then a point on $\Gamma_{\mathrm{c}}^{*}$ with $f<0$ is regarded as to stick on the rigid surface. And the requirement of zero tangential displacement relative to the rigid surface can be satisfied if we stipulate the following relation,

$$
\Delta t_{\mathrm{T} i}=-k_{\mathrm{T}}\left(\Delta u_{\mathrm{T} i}^{\mathrm{e}}-\Delta g_{\mathrm{T} i}\right),
$$

by imposing a large enough penalty parameter, where $\Delta g_{\mathrm{T} i}$ is the prescribed motion of the rigid body. The spring constant $k_{\mathrm{T}}$ may also be properly chosen using experimental works (as discussed by Bowden and Tabor [159]) which support the fact that a small amount of relative displacement is allowed before a point starts to slide.

When the state of stress of a point on $\Gamma_{\mathrm{c}}^{*}$ is such that $f=0$ and $\Delta f=0$, the point is said to be sliding. If an associated isotropic sliding rule,

$$
\Delta u_{\mathrm{T} i}^{\mathrm{P}}=-\Lambda\left(\partial f / \partial t_{\mathrm{T} i}\right),
$$


is adopted to describe the behavior of the sliding (plastic) component $\Delta u_{T i}^{\mathrm{p}}$ of the displacement increment vector $\Delta u_{\mathrm{T}}\left(=\Delta u_{\mathrm{T}} e_{i}\right)$ which is assumed to have the following decomposition:

$$
\Delta u_{\mathrm{T} i}=\Delta u_{\mathrm{T}_{i}}^{\mathrm{e}}+\Delta u_{\mathrm{T} i}^{\mathrm{e}}
$$

The positive scalar function can be obtained from one of the sliding criteria,

$$
\Delta f=\Delta t_{\mathrm{T} i}\left(\partial f / \partial t_{\mathrm{T} i}\right)+\Delta u \mathrm{P}_{\mathrm{T} i}\left(\partial f / \partial \mu_{\mathrm{F}}\right)\left(\partial \mu_{\mathrm{F}} / \partial u_{\mathrm{Ti}}^{\mathrm{P}_{i}}\right)+\Delta t_{n}\left(\partial f / \partial t_{n}\right)=0
$$

and (42), as

$$
\Lambda=\frac{-k_{\mathrm{T}} T_{j}\left(\Delta u_{\mathrm{T}_{i}}-\Delta g_{\mathrm{T}_{j}}\right)+\mu_{\mathrm{F}} \Delta t_{\mathrm{n}}}{k_{\mathrm{T}}+T_{k} t_{n}\left(\partial \mu_{\mathrm{F}} / \partial u_{\mathrm{T} k}^{\mathrm{P}}\right)}
$$

where $\bar{u}_{\mathrm{T}}^{\mathrm{p}}\left(=\sqrt{\overline{u_{\mathrm{T}_{i}}^{\mathrm{P}} u_{\mathrm{T}_{i}}}}\right)$ is the effective sliding displacement, the quantity $\partial \mu_{\mathrm{F}} / \partial \bar{u}_{\mathrm{f}}^{\mathrm{f}}$ is to be $u_{T_{i}}^{\mathrm{p}}$, and $T_{i}$ is defined as

$$
T_{i}=\partial f / \partial t_{T i}=t_{T_{i}} / t_{T}
$$

in which $\bar{t}_{\mathrm{T}}\left(=\sqrt{\boldsymbol{t}_{\mathrm{T}} t_{\mathrm{T}}}\right)$ is termed the effective tangential traction.

Substituting (46) into (43) and using (42) and (44), we arrive at a complete incremental friction law as

with

$$
\Delta t_{\mathrm{T}_{i}}=-k_{i j}\left(\Delta u_{\mathrm{T} j}-\Delta g_{\mathrm{T}_{j}}\right)-c T_{i} \Delta t_{n},
$$

$$
\begin{aligned}
& k_{i j}=k_{\mathrm{T}}\left(\delta_{i j}-\alpha k_{\mathrm{T}} T_{i} T_{j} / D_{\mathrm{T}}\right), \\
& c=\alpha k_{\mathrm{T}} \mu_{\mathrm{F}} / D_{\mathrm{T}}, \\
& D_{\mathrm{T}}=k_{\mathrm{T}}+T_{k} t_{n}\left(\partial \mu_{\mathrm{F}} / \partial \bar{u}_{\mathrm{T}}^{\mathrm{P}}\right)\left(u \mathrm{~T}_{\mathrm{T} k} / \bar{u}_{\mathrm{T}}^{\mathrm{P}}\right),
\end{aligned}
$$

where $\bar{u}{ }_{\mathrm{P}}^{\mathrm{P}}\left(=\sqrt{u_{\mathrm{P}_{i}} u_{\mathrm{T} i}^{\mathrm{P}_{i}}}\right)$ is the effective sliding displacement, the quantity $\partial \mu_{\mathrm{F}} / \partial \bar{u}_{\mathrm{P}}^{\mathrm{P}}$ is to be obtained from the $\mu_{\mathrm{F}}-\bar{u}_{\mathrm{P}}^{\mathrm{P}}$ curve in a simple sliding test such as the experimental work by Courtney-Pratt and Eisner [160] for like-metallic specimens (gold, platinum, tin, indium and mild steel, etc.), and $\alpha=0$ if $f<0$ (sticking) or $f=0$ but $T_{i} \Delta t_{\mathrm{T} i}+\mu_{\mathrm{F}} \Delta t_{n}<0$ (frictional unloading), otherwise $\alpha=1$ when $f=0$ and $T_{i} \Delta t_{\mathrm{T} i}+\mu_{\mathrm{F}} \Delta t_{n} \geqslant 0$ (sliding).

The above incremental form of friction law is obtained based upon the experimental work by Courtney-Pratt and Eisner [160] which shows the existence of small 'elastic' deformations in advance of large plastic sliding. The experimental results resemble plasticity very closely. Detailed explanations of these observations are referred to Bowden and Tabor [159]. One

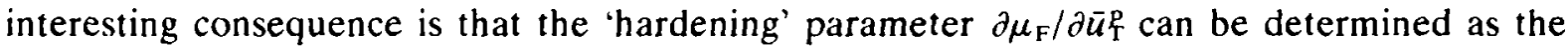
tangent of $\mu_{\mathrm{F}}-\bar{u}_{\mathrm{T}}$ curve obtained from experiments just as $H^{\prime}$ from the stress-logarithmic strain curve in plasticity theory.

Another remark is for the so-called non-local form of friction laws advocated by Oden and Pires [161-162]. If the size of contact surface is too small for the height or the size of asperities 
on the surface of materials to affect the friction, we may replace $t_{n}$ in the incremental friction law (48) by $T_{n}^{*}$ defined as

$$
t_{n}^{*}(y)=\int_{\Gamma^{*}} k(x ; y) t_{n}(y) \mathrm{d} \Gamma(y),
$$

where $k(x ; y)$ is a kernel function satisfying

$$
\int_{\Gamma_{\mathrm{c}}^{*}} \int_{\Gamma_{\mathrm{c}}^{*}} k(\boldsymbol{x} ; \boldsymbol{y}) \mathrm{d} \Gamma(\boldsymbol{y}) \mathrm{d} \Gamma(\boldsymbol{x})=1,
$$

and $k(x ; y)$ becomes almost zero for $\boldsymbol{y}$ with $|\boldsymbol{y}-\boldsymbol{x}|>\boldsymbol{\xi}$. Here $\boldsymbol{\xi}$ is a parameter related to the height and the size of asperities, i.e., the surface roughness. For many applications in metal forming, the size of contact surface is large enough, thus $t_{n}^{*}=t_{n}$ can be assumed. In the following examples, we shall use (48) as the friction law without considering the non-local effect.

\section{Finite element approximation}

Using constitutive equations (32) and (48) with the relation (22), the principle of virtual work of the updated Lagrangian approach (21) including the contact conditions can be rewritten as

$$
\begin{aligned}
& \int_{\Omega^{t}}\left(L_{i j k !} \Delta u_{k, l}+\sigma_{j k}^{t} \Delta u_{i, k}\right) \bar{u}_{i, j} \mathrm{~d} \Omega^{t} \\
& \quad+\int_{\Gamma_{\mathrm{c}}^{t}}\left\{k_{n}\left(\Delta u_{n}-\Delta g_{n}\right) \bar{u}_{n}+\left[k_{i j}\left(\Delta u_{\mathrm{T}_{j}}-\Delta g_{\mathrm{T}_{j}}\right)+c T_{i} \Delta t_{n}\right] \bar{u}_{\mathrm{T} i}\right\} \mathrm{d} \Gamma^{t} \\
& =\int_{\Omega^{\prime}} \rho \Delta b_{i} \bar{u}_{i} \mathrm{~d} \Omega^{t}+\int_{\Gamma_{\mathrm{F}}^{t}} \Delta t_{i} \bar{u}_{i} \mathrm{~d} \Gamma^{t} \quad \forall \bar{u}_{i}\left(=\delta u_{i}\right) \text { such that } \bar{u}_{i}=0 \text { on } \Gamma_{\mathrm{D}}^{t},
\end{aligned}
$$

where $L_{i j k l}=\hat{D}_{i j k l}-\sigma_{i k}^{t} \delta_{j l}-\sigma_{j k}^{t} \delta_{i l}$, and the relation $\Delta t \cdot \bar{u}\left(=\Delta t_{\mathrm{T}} \cdot \bar{u}_{\mathrm{T}}+\Delta t_{n} \bar{u}_{n}\right)$ has been used.

Finite element approximations to the integral form (54) follow standard procedures [163] by discretizing the domain $\Omega^{t}$ and the boundary $\Gamma^{t}$ into finite elements $\Omega_{e}^{t}$ and $\Gamma_{e}^{t}$. Within each element we interpolate the increment of displacement by the shape functions $\left\{N_{\alpha}(x)\right\}$ as

also

$$
\Delta u_{i}(x)=\Delta u_{i \alpha} N_{\alpha}(x)
$$

$$
\bar{u}_{i}(x)=\bar{u}_{i \alpha} N_{\alpha}(x),
$$

where $\alpha$ is the local node number ranging from 1 to the total number of nodes in one element. $\Delta u_{i \alpha}$ and $\bar{u}_{i \alpha}$ are the $i$ th components of the displacement-and virtual displacement-increment vectors at $\alpha$ th node, respectively.

Substituting (55) and (56) into (54), using the arbitrariness of $\bar{u}_{i}$, we have 


$$
\left\{\sum_{e} K_{i j \alpha \beta}^{\Omega_{e}}+\sum_{e} K_{i j \alpha \beta}^{\Gamma_{e e}}\right\} \Delta u_{j \beta}=\sum_{e} f_{i \alpha}^{\Omega_{e}}+\sum_{e} f_{i \alpha}^{\Gamma_{\mathrm{Fe}}}+\sum_{e} f_{i \alpha}^{\Gamma_{\mathrm{ce}}}
$$

where

$$
\begin{aligned}
& K_{i j \alpha \beta}^{\Omega_{e}}=\int_{\Omega_{e}^{\prime}}\left\{\frac{1}{2}\left(L_{i k j l}+L_{i k l j}\right)+\delta_{i j} \sigma_{k l}^{\prime}\right\} N_{\alpha k} N_{\beta l} \mathrm{~d} \Omega^{t}, \\
& K_{i j \alpha \beta}^{\Gamma_{c e}}=\int_{\Gamma_{\mathrm{c} e}^{*}}\left\{k_{n} n_{i} n_{j}+\hat{k}_{i j}\right\} N_{\alpha} N_{\beta} \mathrm{d} \Gamma^{t},
\end{aligned}
$$

are the element stiffness matrices, and

$$
\begin{aligned}
& f_{i \alpha}^{\Omega_{e}}=\int_{\Omega_{e}^{t}} \rho \Delta b_{i} N_{\alpha} \mathrm{d} \Omega^{t}, \quad f_{i \alpha}^{\Gamma_{\mathrm{Fe}}}=\int_{\Gamma_{\mathrm{Fe}}^{t}} \Delta t_{i} N_{\alpha} \mathrm{d} \Gamma^{t}, \\
& f_{i \alpha}^{\Gamma_{\mathrm{ce}}}=\int_{\Gamma_{\mathrm{ce}}^{t}}\left\{k_{n} \Delta g_{n} n_{i}+\left(k_{i k} \Delta g_{\mathrm{T} k}+c T_{i} \Delta t_{n}\right)\right\} N_{\alpha} \mathrm{d} \Gamma^{t},
\end{aligned}
$$

are the element load vectors, $\hat{k}_{i j}=k_{i j}-k_{k j} n_{i} n_{k}-k_{i k} n_{j} n_{k}+k_{k l} n_{i} n_{j} n_{k} n_{l}$, and the assembling summations $(\Sigma)$ are performed over the total numbers of elements in $\Omega^{t}, \Gamma_{\mathrm{F}}^{t}$, and $\Gamma_{\mathrm{c}}^{t}$, respectively.

Equation (57) is a system of linear equations provided that the contact pressure $t_{n}$ remains constant. However, if $\Delta t_{n} \neq 0$ and is quite different from the previous step, then an iteration scheme must be introduced when the frictional case is considered.

\section{Numerical examples}

The following example problems are intended to verify the formulations given in this article and to show the capability of the computer program 'EPAFRIC' developed. Emphases are placed on the investigation of numerical accuracy of finite element solutions to nonlinear large deformation elastic-plastic problems.

It is well known that for elastic-plastic materials the response of finite elements is too stiff in the fully plastic range. This is due to the requirement of vanishing plastic strain increment $\left(\Delta \varepsilon_{i i}^{\mathrm{p}}=0\right)$ in the constitutive equations, although with strain hardening part of the strain increment is elastic. The nearly incompressible deformation severely restricts the selection of types of finite elements available. The behaviors of many different elements were carefully examined by Nagtegaal et al. [86]. A special arrangement of a quadrilateral element which consists of four constant strain triangles obtained by connecting its diagonals was proved to be free of overconstraint for plane-strain problems. This model is used in the following analysis.

\subsection{Necking in a simple tension test}

This problem has been solved by Needleman [29], McMeeking and Rice [27], Argyris et al. [56-58], Kikuchi and Cheng [61], and Chen [43] using finite element methods, also by Chen [164], Norris et al. [165], and Saje [166] using finite difference methods. In the present work, a cylindrical bar of an initial length $2 l_{0}=12 \mathrm{in}$. and an initial radius $r_{0}=1 \mathrm{in}$. as shown in Fig. 4 is 


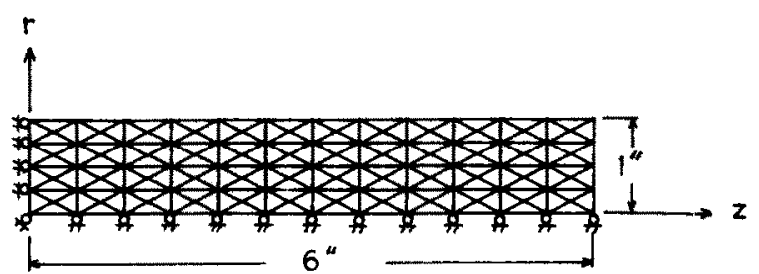

(a)

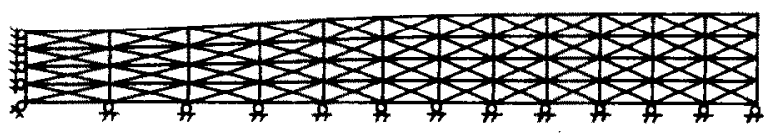

(b)

Fig. 4. (a) An initial mesh for a uniaxial tension test. (b) The deformed mesh at $30 \%$ elongation. Material constants: $E=30 \times 10^{6} \mathrm{psi} ; \nu=\frac{1}{3} ; S_{Y}=60 \mathrm{ksi} ; \bar{\sigma}=120 \bar{\varepsilon}^{0.125} \mathrm{ksi}$.

used to perform this test. The material is mild steel whose property is characterized by: Young's modulus $E=30 \times 10^{6} \mathrm{psi}$, Poisson's ratio $\nu=\frac{1}{3}$, yield strength $S_{Y}=60 \mathrm{ksi}$. The postyield stress-strain curve is described by

$$
\bar{\sigma}=120 \bar{\varepsilon}^{0.125} \mathrm{ksi},
$$

where $\bar{\varepsilon}\left(=\sqrt{2 e_{i j} e_{i j}}\right)$ is the effective strain, $e_{i j}\left(=\varepsilon_{i j}-\frac{1}{3} \delta_{i j} \varepsilon_{k k}\right)$.

In order to initiate the bifurcation, an initial imperfection of $1 \%$ of the radius is introduced artificially on the central portion of the bar. Due to symmetry, one quarter of the specimen is modelled by 192 finite elements with 226 degrees of freedom. 300 displacement increments are taken to complete this analysis up to $30 \%$ elongation (Fig. 4). \% elongation (engineering strain) is defined as $100 \% \cdot\left(l-l_{0}\right) / l_{0}$, where $l$ is the current length and $l_{0}$ the initial length of the bar. Plastic unloading begins at $15.9 \%$ elongation and propagates in the specimen. Since then local necking on the center of the bar becomes severe. The development of neck as a function of elongation is depicted in Fig. 5. This localized phenomenon is measured either by the radial displacement at the neck or by the ratio $A / A_{0}$ where $A$ is the deformed area of the

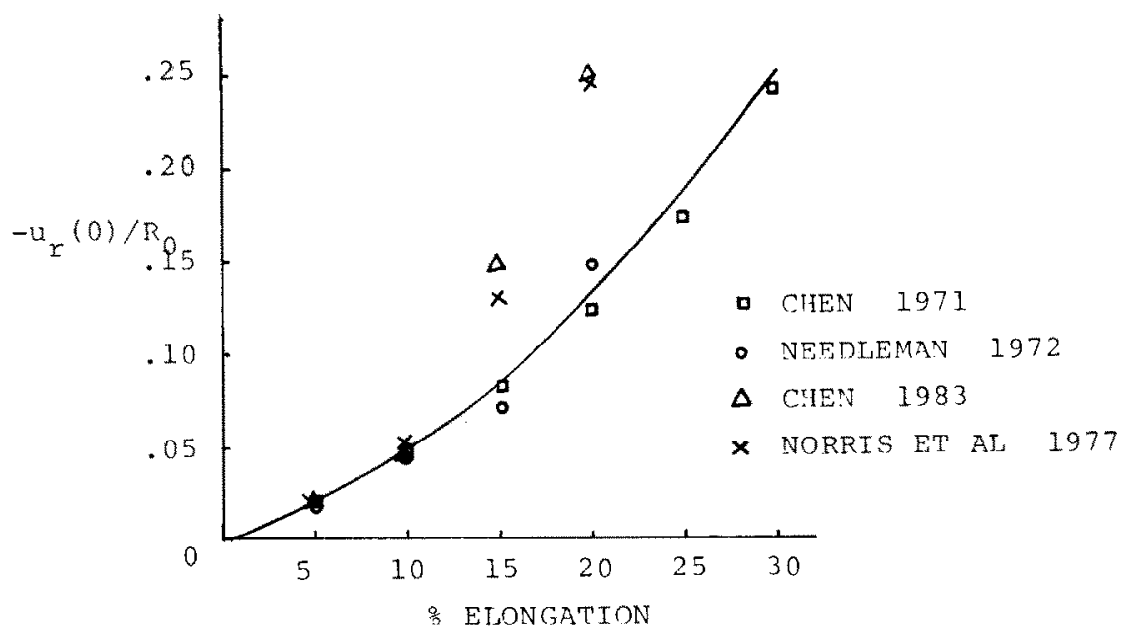

Fig. 5. Development of neck as a function of $\%$ elongation; $u_{r}(0)=$ radial displacement at neck, $R_{0}=$ initial radius. 
neck and $A_{0}$ is the initial area. Numerical solutions obtained by Chen [164], Needleman [29], Norris et al. [165] and Chen [43] are included to compare with the present calculations. The border lines between the unloading and the plastic loading regions are shown in Fig. 6 at three different deformation stages. As also observed by Chen [164], once the unloading occurs, instead of the contraction of the area, the radius at the end expands slightly as a result of the decrease of the axial stress.

Fig. 7 illustrates the load-deformation curve. The applied load is normalized by the maximum which is $273 \times 10^{3} \mathrm{lbs}$. at $11 \%$ elongation in this analysis. The maximum load is achieved when the rate of decrease of cross-sectional area reaches the rate of hardening effect. Again comparisons are made with other investigators. They show fairly good agreement despite that different postyield stress-strain relations are used. The dotted line in the figure is another solution obtained from a different discretization shown in Fig. 8. It is apparent that

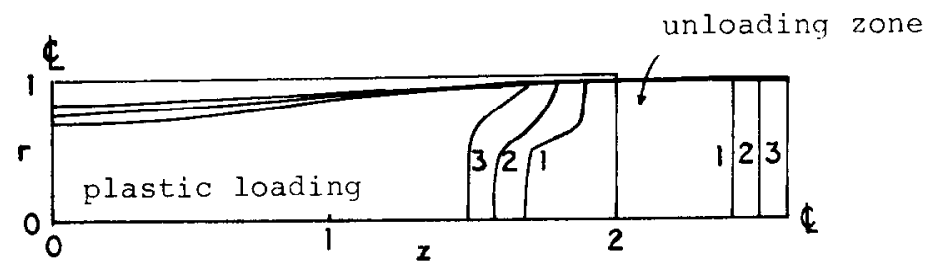

Fig. 6. Development of unloading zone and neck. 1: 20\% elongation; $2: 25 \%$ elongation; $3: 30 \%$ elongation.

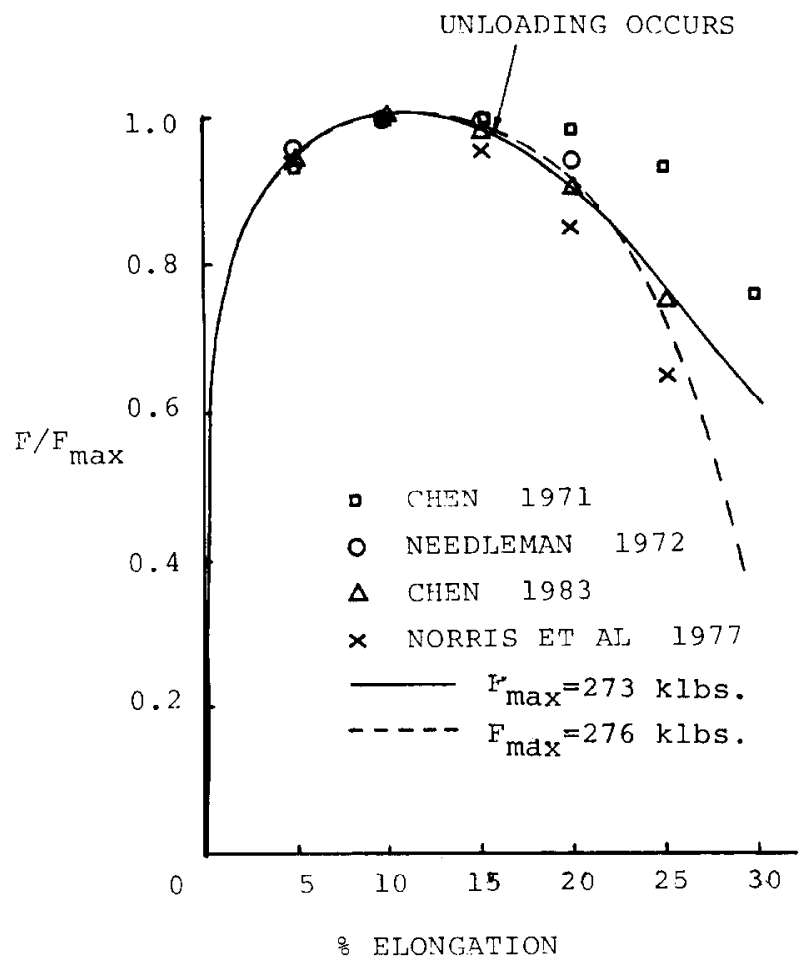

Fig. 7. Normalized applied load vs. engineering axial strain; $F_{\max }=\operatorname{maximum~load.~}$ 


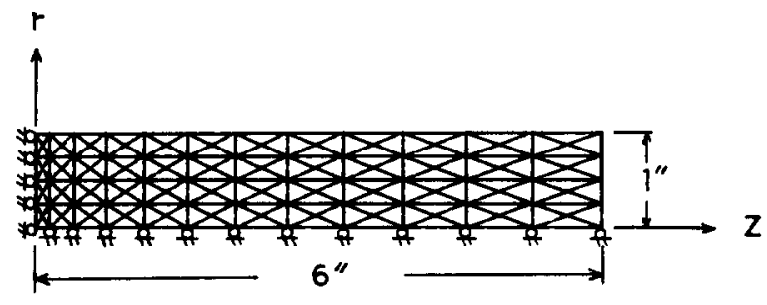

(a)

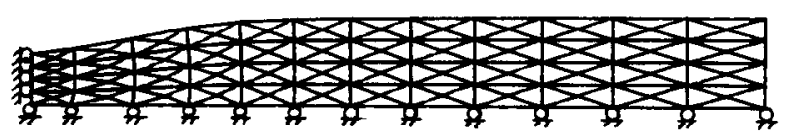

(b)

Fig. 8. (a) An initial mesh for a uniaxial tension test. (b) The deformed mesh at $30 \%$ elongation. Material constants: $E=30 \times 10^{6} \mathrm{psi} ; \nu=\frac{1}{3} ; S_{Y}=60 \mathrm{ksi} ; \bar{\sigma}=120 \bar{\varepsilon}^{0.125} \mathrm{ksi}$.

both the predicted load and the deformation strongly depend on finite element discretizations. Thus for nonlinear problems the choice of a proper initial finite element mesh becomes a very important aspect. The question of what kind of model provides the best solution is, however, unknown yet. The stress distributions at the neck are shown in Fig. 9 for the axial stress $\sigma_{z z}$ and the mean stress $\sigma_{\mathrm{m}}$ as a function of $r / R$, where $R$ is the current radius of the neck and $r$ is measured from the center line. For comparison purposes, the data are chosen from different sources at approximately the same neck radius and are normalized by the initial yield strength.

To see the behavior of finite elements, the computed stress-strain curves for two typical elements are given in Fig. 10. The longer curve is for the one at the middle and the other is for the one at the end of the bar. The decrease of the effective stress accounts for the effect of unloading. The element becomes almost rigid after unloading occurs.

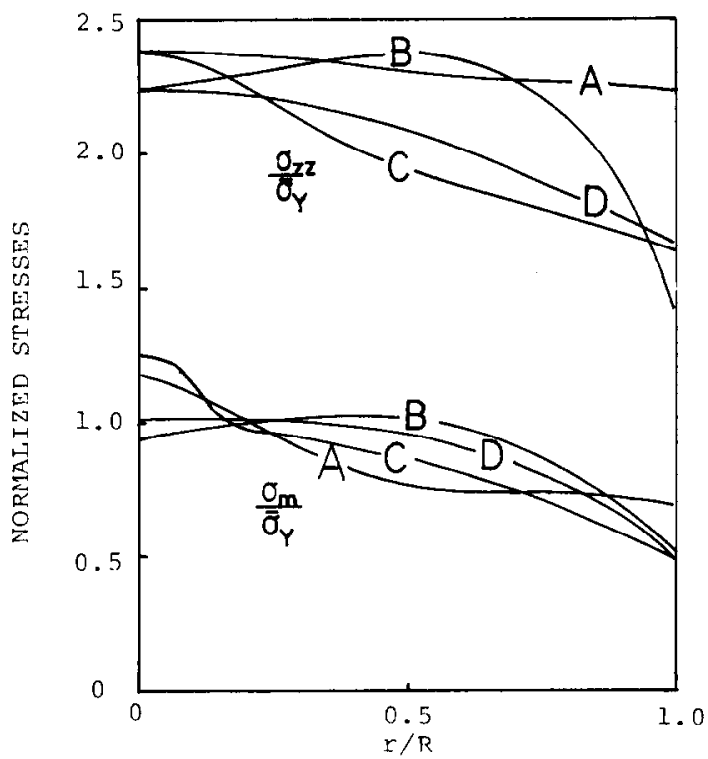

Fig. 9. Comparison of axial $\left(\sigma_{z z}\right)$ and mean $\left(\sigma_{\mathrm{m}}\right)$ stress distributions at neck, $\bar{\sigma}_{Y}$ is the initial yield strength. A: present result at $R / R_{0}=0.744$; B: Chen [164] at $R / R_{0}=0.764$; C: Needleman [29] at $R / R_{0}=0.769$; C: Norris et al. $[165]$ at $R / R_{0}=0.775$. 


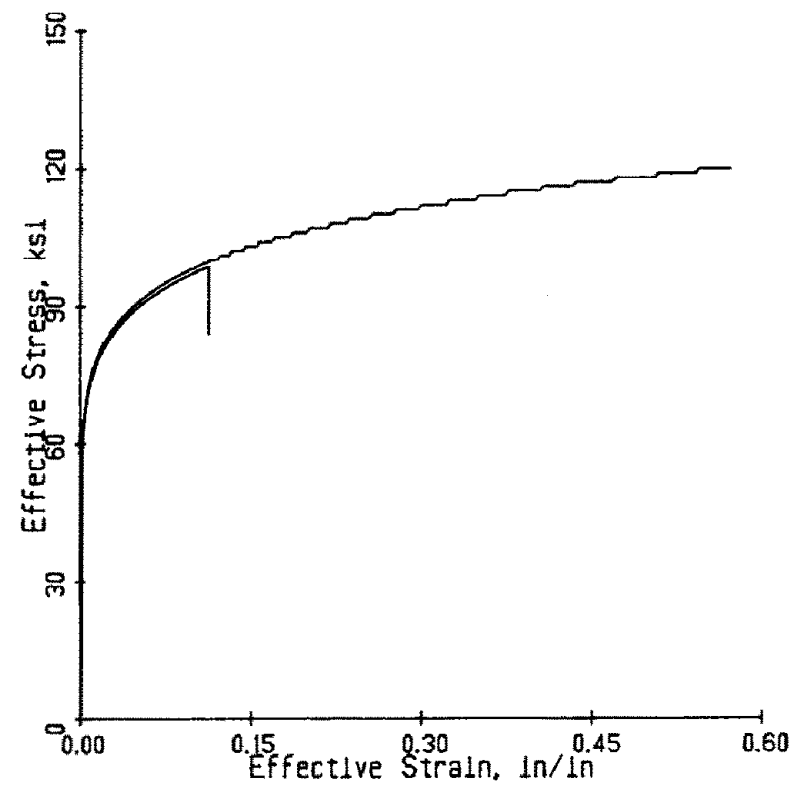

Fig. 10. Computed effective stress-strain curves for two elements in a uniaxial tension test.

\subsection{Upsetting of a cylindrical block}

The next example problem is the upsetting of a cylindrical block shown in Fig. 11. The block has an initial diameter of $20 \mathrm{~mm}$ and height of $30 \mathrm{~mm}$. The mechanical properties and the hardening effect of the material are specified as follows: Young's modulus $E=$ $200 \mathrm{kN} / \mathrm{mm}^{2}$, Poisson's ratio $\nu=0.3$, initial yield strength $S_{Y}=0.7 \mathrm{kN} / \mathrm{mm}^{2}$, strain hardening
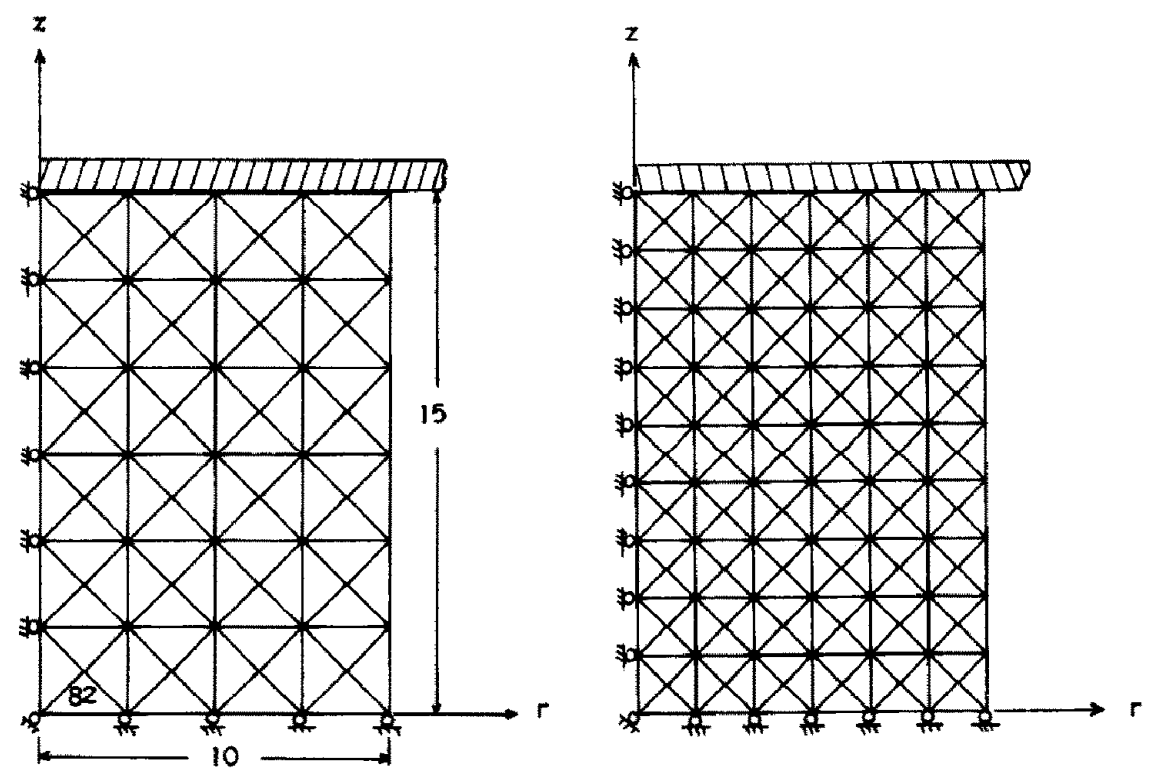

Fig. 11. Initial finite element meshes for an upsetting analysis. 
$H^{\prime}=0.3 \mathrm{kN} / \mathrm{mm}^{2}$. A quarter of the block is analyzed due to the symmetry of the problem. Several cases of different frictional conditions along the punch-billet interface are solved. Fig. 12 illustrates the computed results for the upsetting load as a function of the reduction in height of the block. The load is computed from the stresses of $\sigma_{z z}$ in the elements adjacent to the interface. For the case of frictionless interface, the solution is found to coincide with the exact one, $\pi R^{2} \bar{\sigma}$, where $R$ is the current radius and $\bar{\sigma}$ the current yield strength of the material. Also included in the figure are results extracted from a joint examination project summarized by Kudo and Matsubara [49], and the works of Yamada et al. [46] and Taylor [60]. They are all based on the same material constants given above, and an assumption of perfectly rough, i.e., sticking interface. An incremental scheme with Newton's iterative method was used by Taylor, while Yamada et al. adopted an approach including two load correction vectors, one for the nodal force equilibrium and the other for the overshooting of stress values due to the violation of yield condition.

It is found that the solutions depend upon the choice of the finite element discretization and the size of increment to some extent. This can be seen from the upsetting loads obtained by choosing the increments of $0.5 \%$ and of $0.25 \%$ with the finite element model of Fig. 11(a), and $0.25 \%$ increment with the mesh depicted in Fig. 11(b). The evergoing over-estimate of the upsetting load (curve A, Fig. 12) for the case of $0.5 \%$ increment can also be observed from Fig. 13 in which the effective stress-strain are evaluated for the element 82 shown in Fig. 11(a).

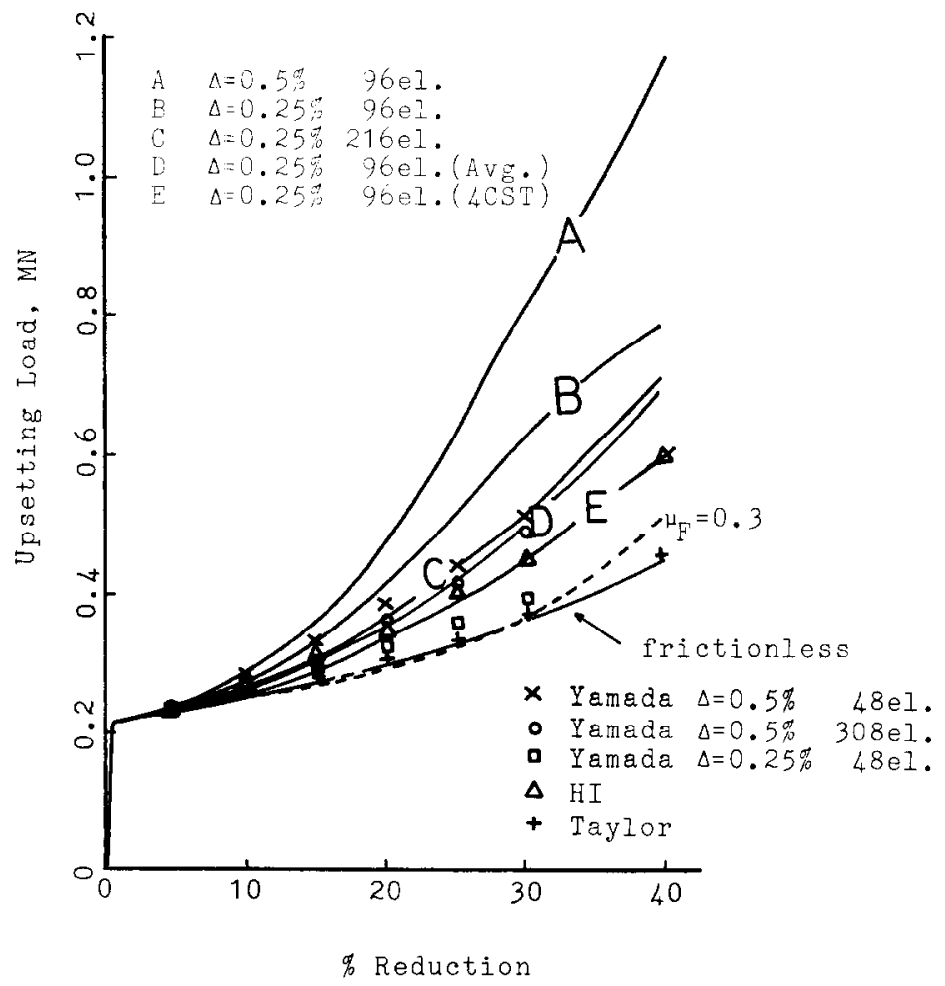

Fig. 12. Upsetting load vs. \% reduction. 


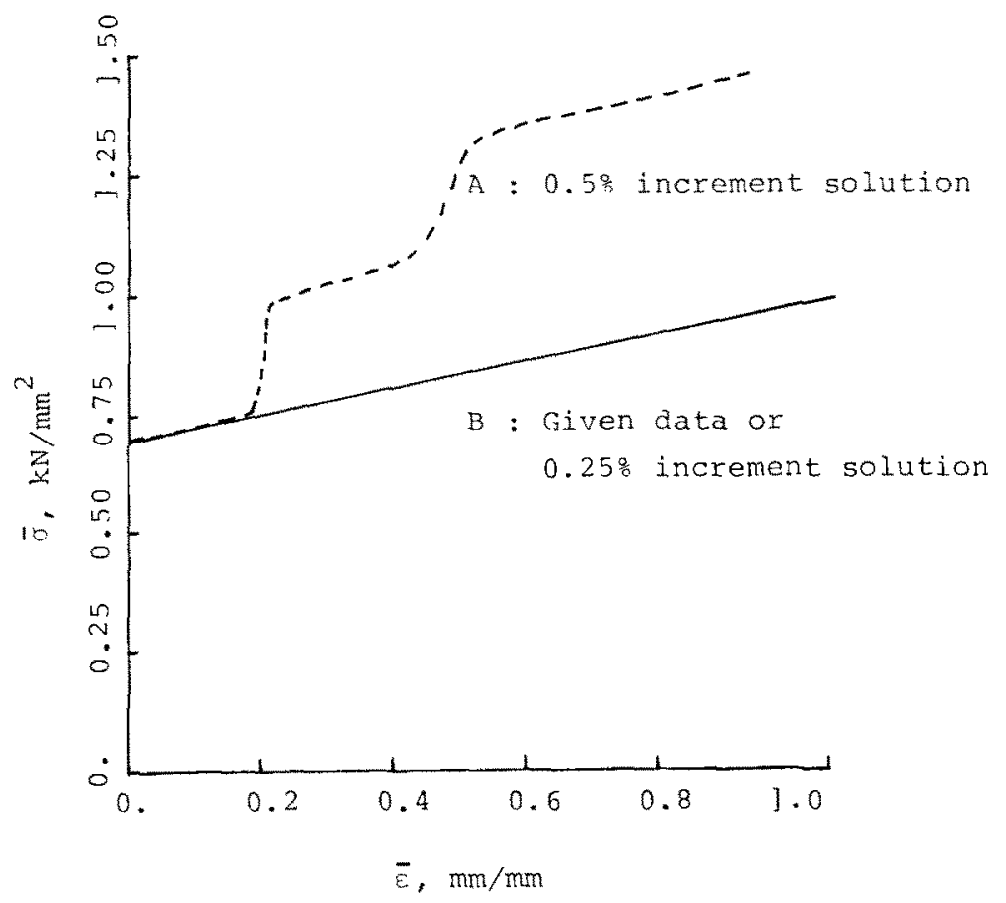

Fig. 13. Effective stress-strain curves in the element 82 of Fig. 11.

The response of this element follows very closely to the given material properties for the case of $0.25 \%$ increment, but, deviates considerably from the expected curve when $0.5 \%$ increment is used. Similar results have also been concluded by Yamada et al. While the improvement of the solution seems to be so profound by reducing the increment size from $0.5 \%$ to $0.25 \%$, taking increments smaller than $0.25 \%$ with the same mesh discretization does not give any change of the result. A finer mesh as in Fig. 11(b) does, however, show much decrease of the predicted upsetting load (curve C, Fig. 12).

Two deformed meshes at $40 \%$ reduction obtained by $0.25 \%$ increment are shown in Fig. 14(a). The severe distortion of finite elements which is not found in the tension test, is due to the sticking constraint on the top surface of the block. It is because of this additional constraint that the so-called locking phenomenon arises which is also revealed in the computed upsetting loads at the stages of larger deformation. This effect can also be observed from the zig-zag pattern in the deformed mesh. In order to overcome this overconstraint, a remedy is to take averages of stresses and strains for each four elements within one quadrilaterial. The results are demonstrated by a drop in the upsetting load (curve D in Fig. 12) and a much smoother deformation pattern (Fig. 14(b)). The more restricted deformation of those two shaded elements is because that they become stiffer by having higher stress values contributed from the other two who are in the dead zone. Another consideration is to artifically reconnect the diagonals of each quadrilateral for every updated finite element mesh. This results in a very soft response as indicated in curve E, Fig. 12 . The deformed mesh at $40 \%$ reduction is shown in Fig. 14(c). A similar deformation pattern has been obtained by Nagtegaal and De Jong [47]. Properties of the 4CST element were studied in details by Kikuchi [167]. 

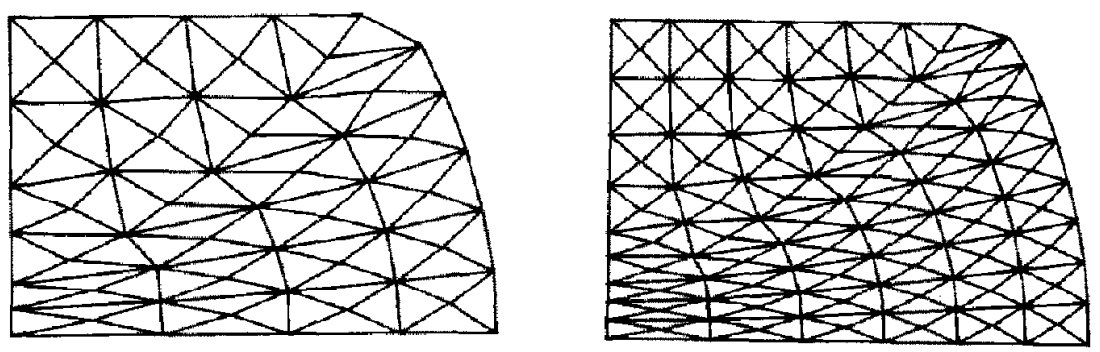

(a)

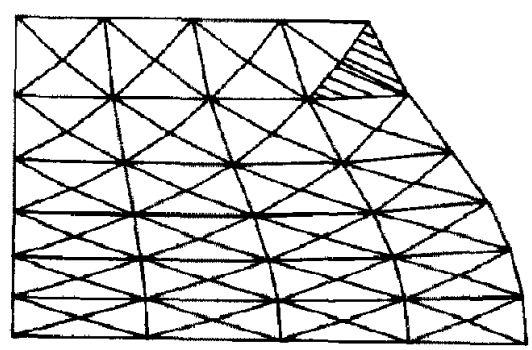

(b)

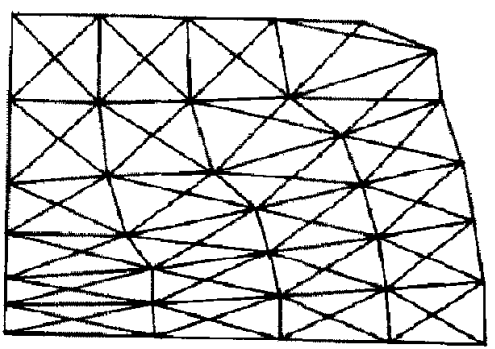

(c)

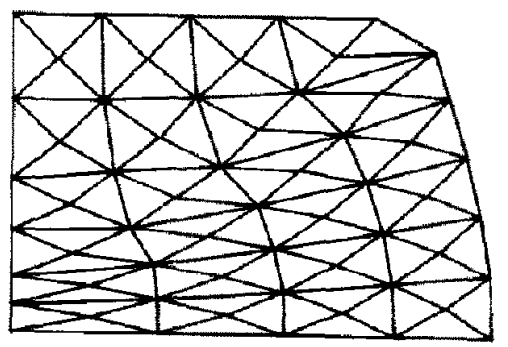

(d)

Fig. 14. Deformed meshes at $40 \%$ reduction of height; sticking punch-billet interface assumed in (a)-(c), $\nu_{\mathbf{F}}=0.3$ in (d).

It is worthwhile to emphasize more on the importance of solution dependence on finite element discretizations. Fig. 15 shows two other initial meshes and corresponding deformed configurations at $40 \%$ reduction. The computed applied loads are illustrated in Fig. 16 which also includes the results reproduced from curve $B$ and curve $C$ in Fig. 12 for comparison.

Removing the restriction of perfectly rough interface, the analysis is also repeated for a frictional case with a coefficient friction $\mu_{\mathrm{F}}=0.3$ assumed to be constant. A less distorted mesh 

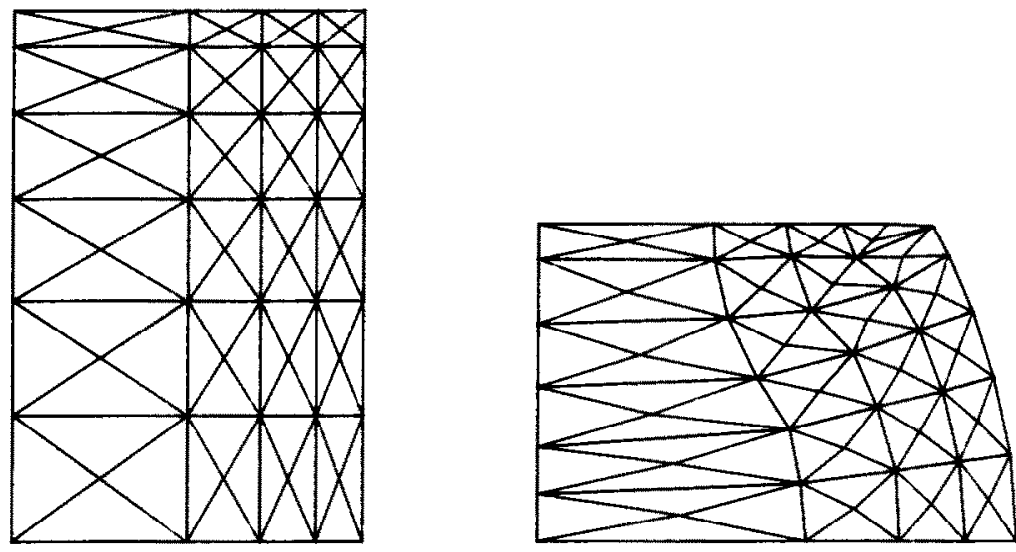

(a)
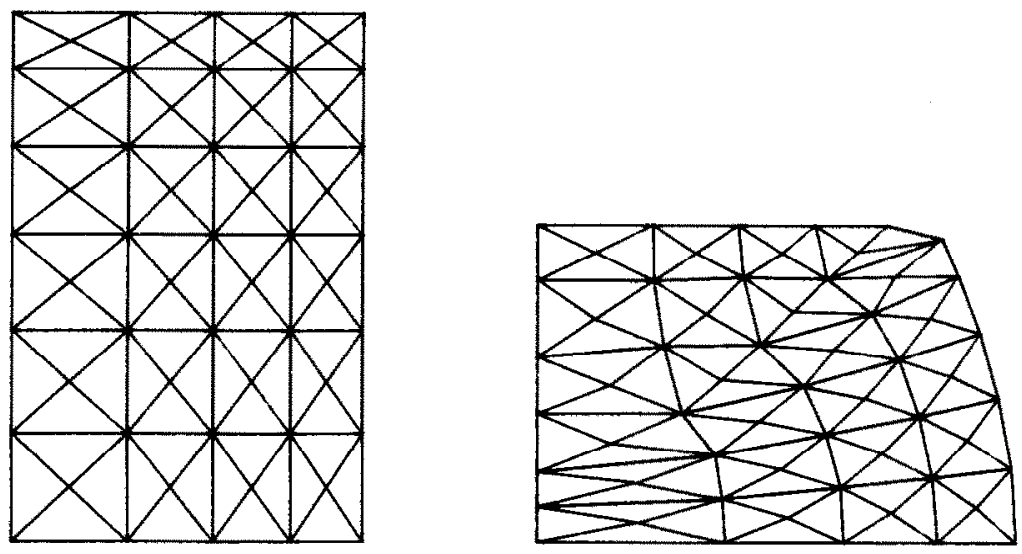

(b)

Fig. 15. Two different finite element discretizations for an upsetting process with sticking interface.

than those in the sticking case is shown in Fig. 14(d). It is noted that between $15 \%$ and $30 \%$ reductions, the upsetting load (Fig. 12) is a little bit smaller than that of the friction-free case. This is because that in the frictionless case, the increase of the block radius (which is refrained when $\mu_{F}=0.3$ ) surpasses the rate of strain hardening.

The equatorial stresses $\sigma_{z z}$ and $\sigma_{\theta \theta}$ obtained from the outermost element at the middle as a function of $\%$ reduction are shown and compared in Fig. 17 for three cases with others' results. The ever-decreasing values of $\left|\sigma_{z z}\right|$ suggest that a much smaller upsetting load at the later stage of compression will be obtained if the load is evaluated on the equatorial plane although its area is increasing. 


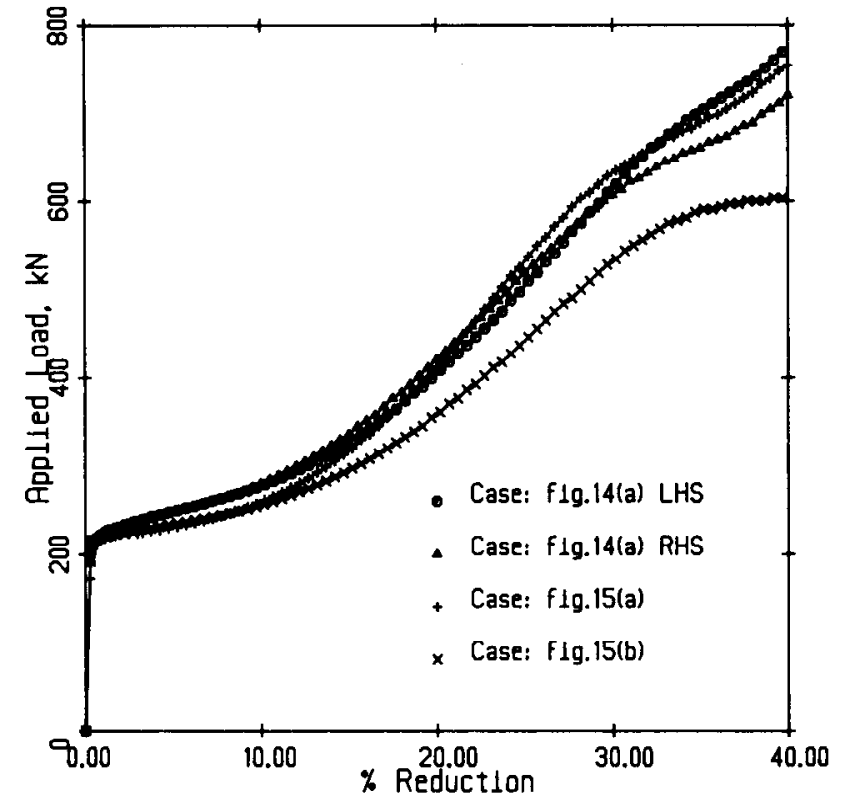

Fig. 16. Comparison of applied loads for an upsetting problem with four different finite element discretizations, sticking interface, $0.25 \%$ disp. increments.

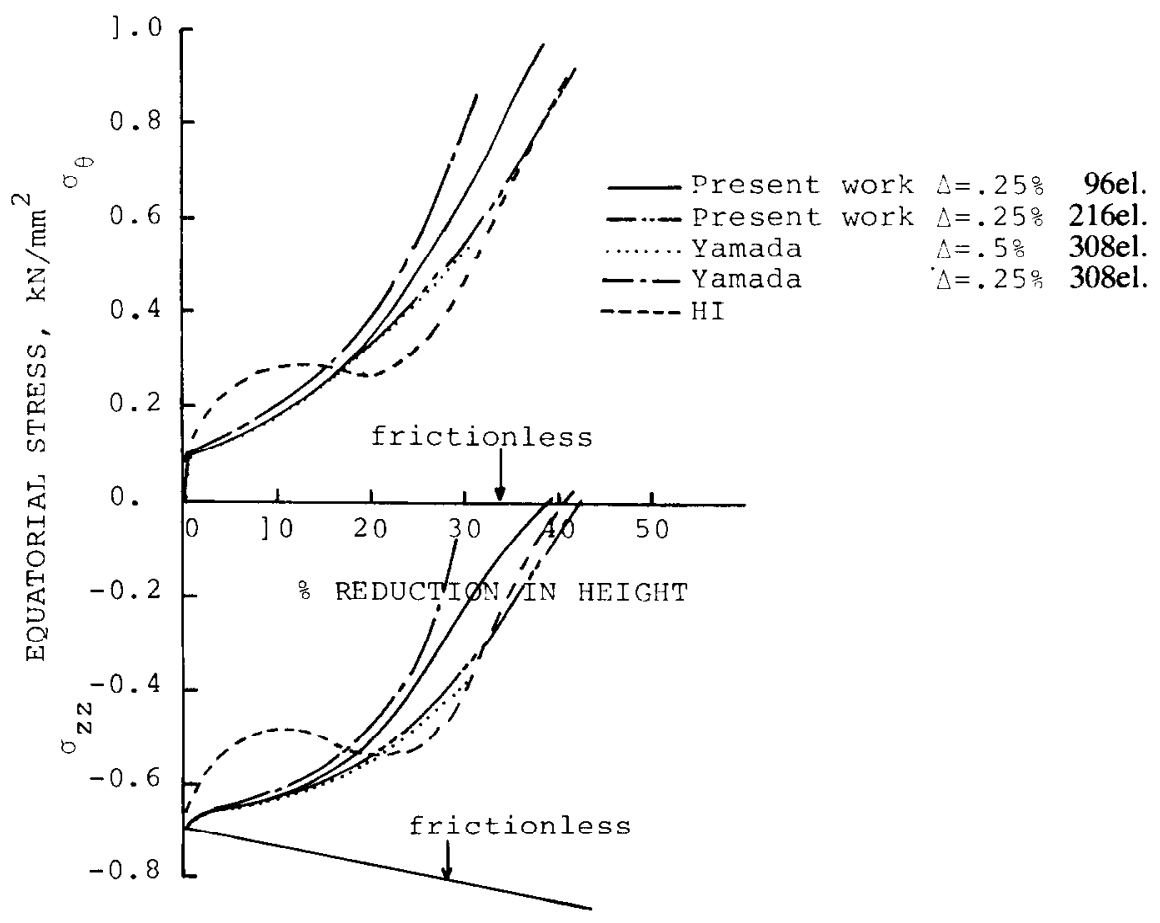

Fig. 17. Comparison of equatorial stresses, $\sigma_{\theta}$ and $\sigma_{z z}$, as a function of $\%$ reduction in height. 


\subsection{An indentation problem}

This example is aimed to test the friction algorithms proposed in this paper. The problem chosen is an indentation analysis of a spherical rigid punch on a cylindrical elastic-plastic block (axisymmetrical case) whose dimensions of the cross-section with a finite element model are shown in Fig. 18. The radius of the punch is $8 \mathrm{~cm}$. Similar analyses have been carried out by Lee and Kobayashi [168], Hardy, Baronet and Tordion [169], Dumas and Baronet [170], Lee, Masaki and Kobayashi [171], and Kikuchi and Skalski [97] under the frictionless condition.

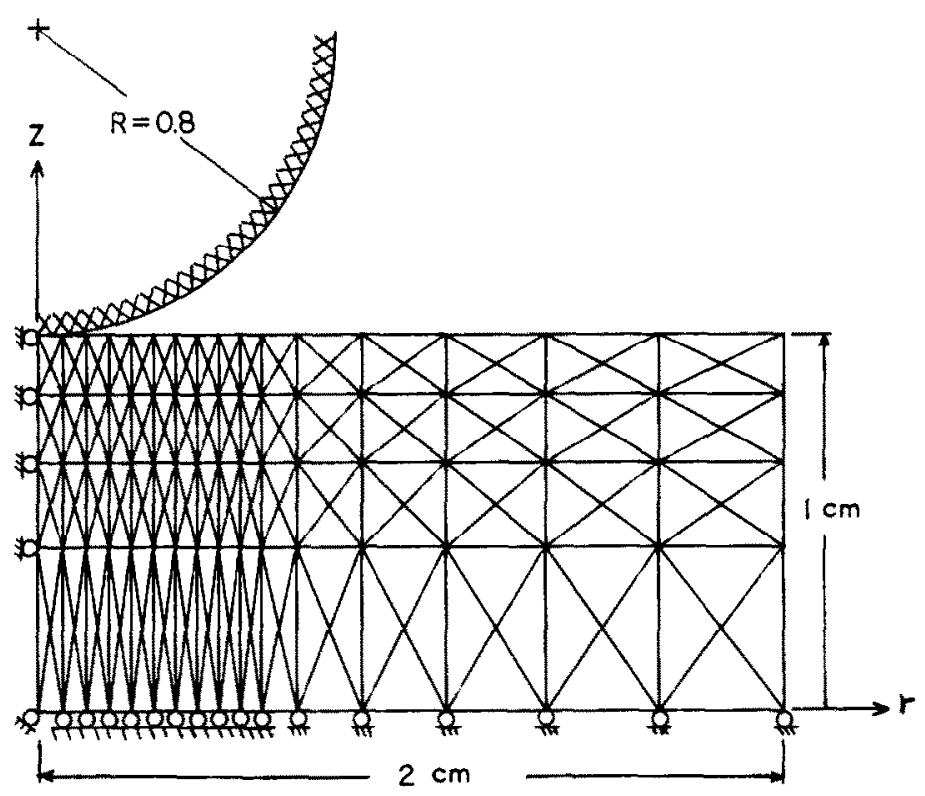

Fig. 18. An indentation problem of a rigid sphere into a deformable cylinder (axisymmetric case).

Material properties of the deformable body are assumed to be Young's modulus $E=$ $1000 \mathrm{kN} / \mathrm{cm}^{2}$, Poisson's ratio $\nu=0.3$, initial yield strength $S_{Y}=10 \mathrm{kN} / \mathrm{cm}^{2}$, and a hardening rule of $\bar{\sigma}=10+5(\bar{\varepsilon}-0.01) \mathrm{kN} / \mathrm{cm}^{2}$. The problem is first solved by assuming elastic deformations only. A reference displacement increment of $0.05 \mathrm{~cm}$ of the punch motion is prescribed unless it violates either the kinematic contact condition (36) or the Coulomb slip criterion (41). Fig. 19 illustrates the computed contact stresses with deformed meshes at $0.4 \mathrm{~cm}$ indentation for three different coefficients of friction. The contour lines on the left-half part of the block represent the distribution of equi-effective stress $\bar{\sigma}$. It is seen that the result of smooth indentation $\left(\mu_{F}-0\right)$ is in good agreement with the Hertz's solution. The peak values of the tangential traction separate the sticking and sliding zones of the contact surface. Similar results have also been presented by Kikuchi [172] who solved the same problem using an iterative method.

Allowing plastic deformations, the problem is again solved for two different coefficients of friction. The results at $0.4 \mathrm{~cm}$ indentation are shown in Fig. 20. In this computation, the reference increment is chosen to be $0.005 \mathrm{~cm}$ which may be changed by the yield criterion (27) besides those two conditions mentioned above. 


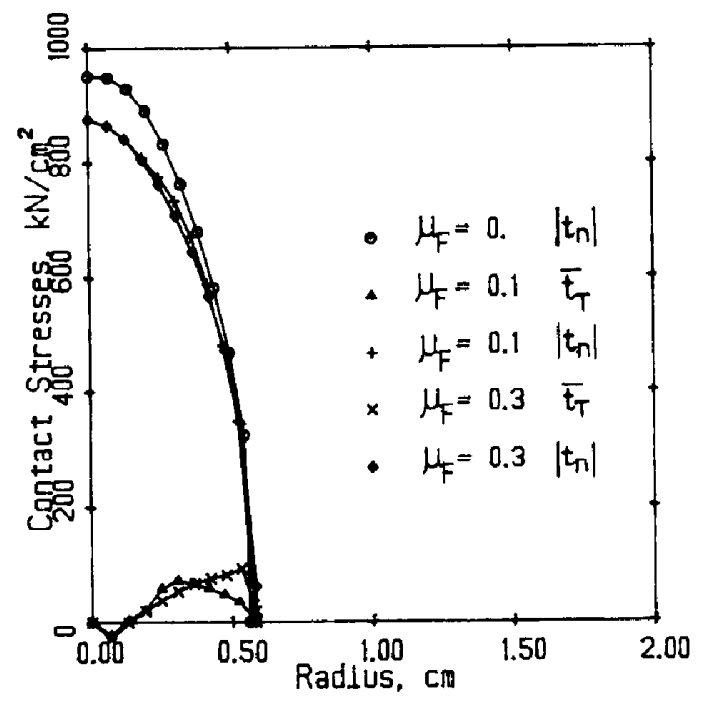

$\mu_{F}=0$.

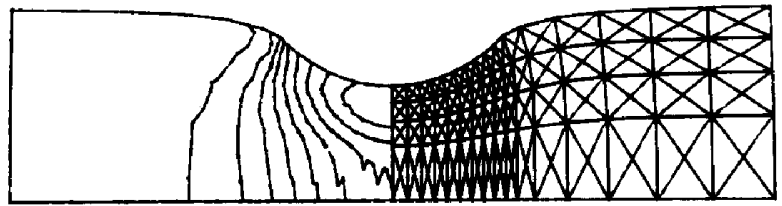

$\mu_{F}=0.1$

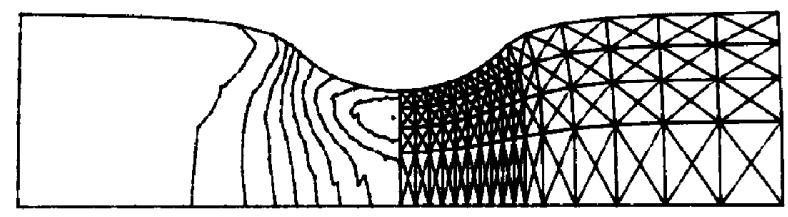

$\mu_{F}=0.3$

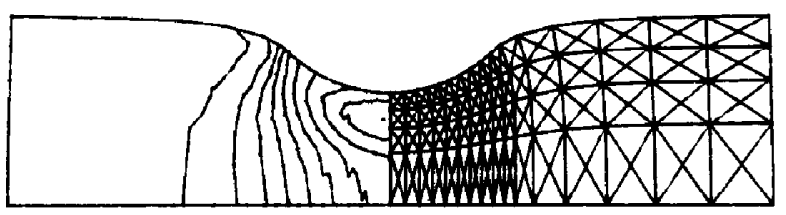

Fig. 19. Contact stresses and deformed meshes at $0.4 \mathrm{~cm}$ indented depth for an elastic indentation test. Material constants: $E=10 \mathrm{Gpa} ; \nu=\mathbf{0 . 3}$. 


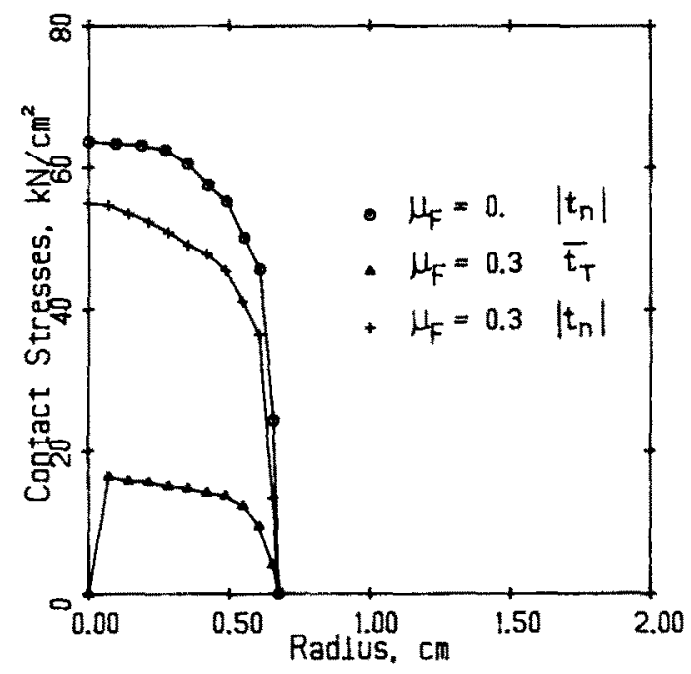

$$
\mu_{\mathrm{F}}=0
$$

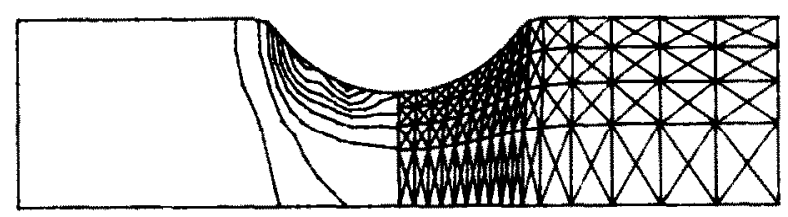

$$
\mu_{F}=0.3
$$

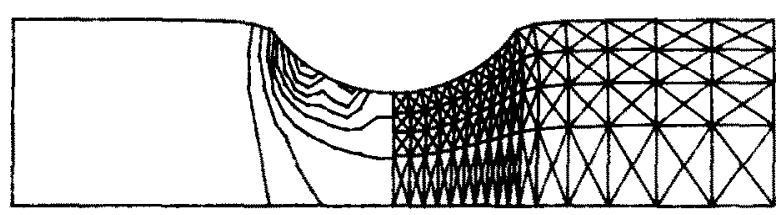

Fig. 20. Contact stresses and deformed meshes at $0.4 \mathrm{~cm}$ indented depth for an axisymmetric indentation test on an elasto-plastic material. Material constants: $E=10 \mathrm{Gpa} ; \nu=0.3 ; S_{Y}=100 \mathrm{Mpa} ; \bar{\sigma}=100+50(\bar{\varepsilon}-0.01) \mathrm{Mpa}$.

\subsection{A head forming process}

As the last example, let us consider a head forming problem with an initial configuration and material properties shown in Fig. 21. The same problem has been solved by Argyris et al. 


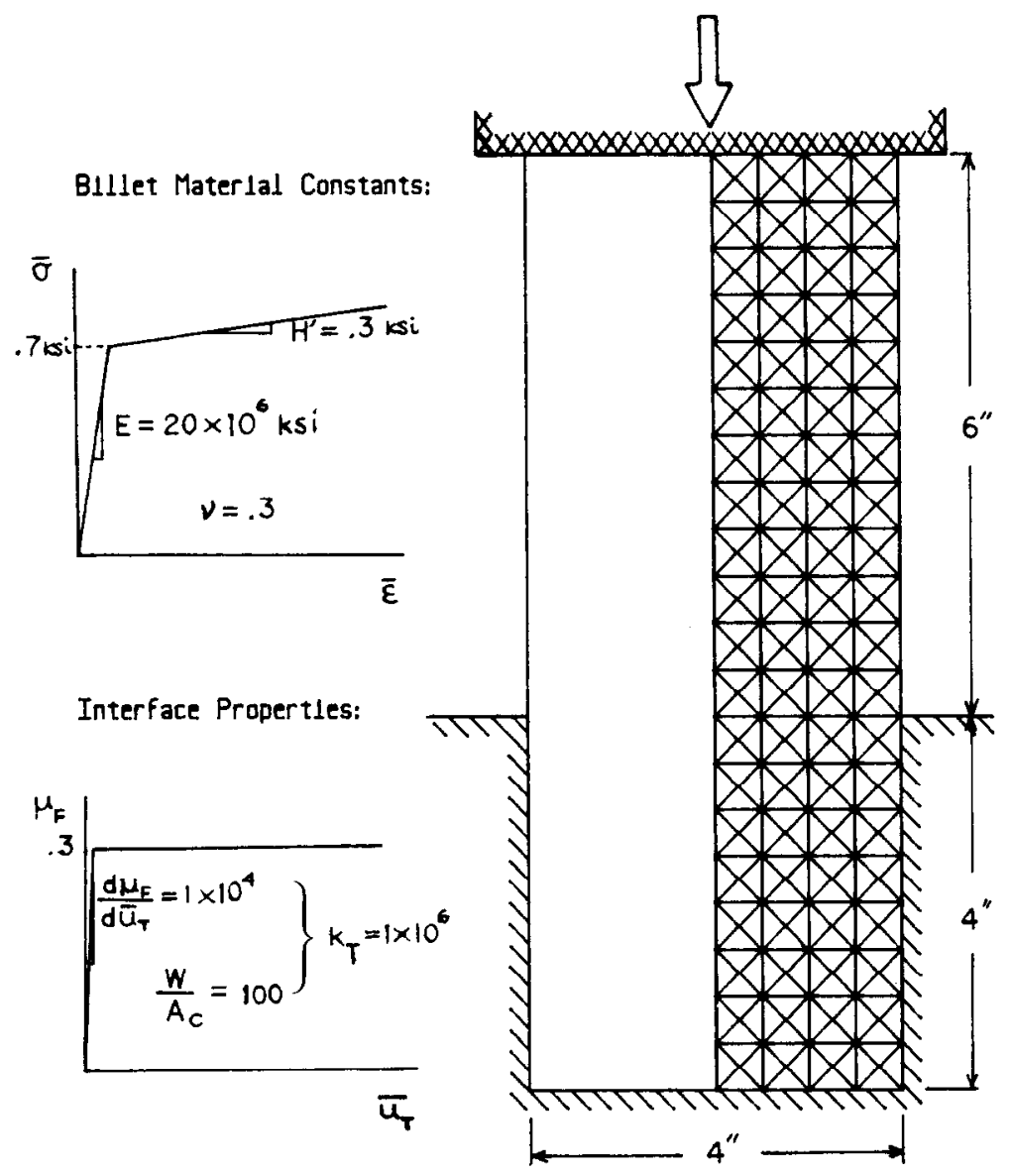

Fig. 21. Initial configuration for a head forming problem.

[59] using both the viscoplastic flow model and the updated Lagrangian model with NewtonRaphson corrections, and by Kobayashi [69] using the rigid-plastic approach.

The analysis is carried out under a reference displacement increment of $0.25 \%$ of the initial height. Deformed configurations at various stages are displayed in Fig. 22 together with the contour lines of the effective stress $\bar{\sigma}$. Localized plastic deformations in the core of the head grow into two shearing bands in the upper and lower parts of the head. Fig. 23 shows the historical path of the applied load evaluated from element stresses under the punch. The slight disturbance at $36 \%$ reduction amounts to the response of the material when some portions of the initially vertical lateral surface of the billet come into contact with the die and the punch (see the deformed mesh at $40 \%$ reduction in Fig. 22). 
100

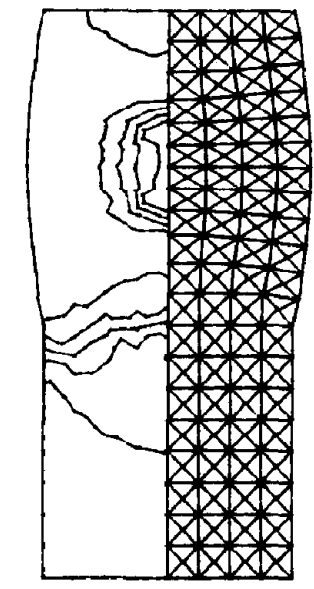

$10 \%$ Reduction

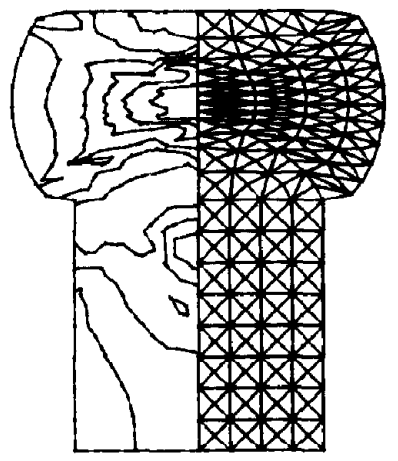

30\% Reduction

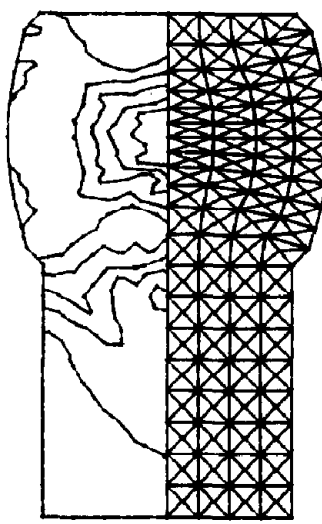

20\% Reduction

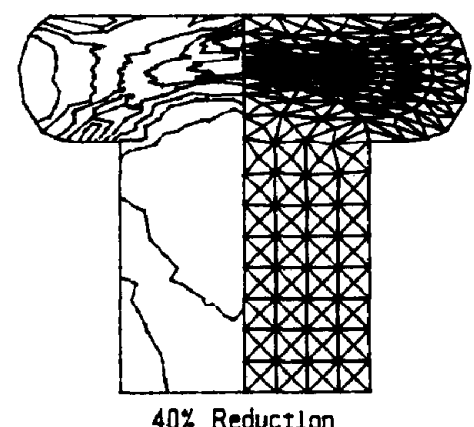

40\% Reduction

Fig. 22. Deformed meshes of a head forming problem by using material constants $\varepsilon$ interface properties shown in Fig. 21.

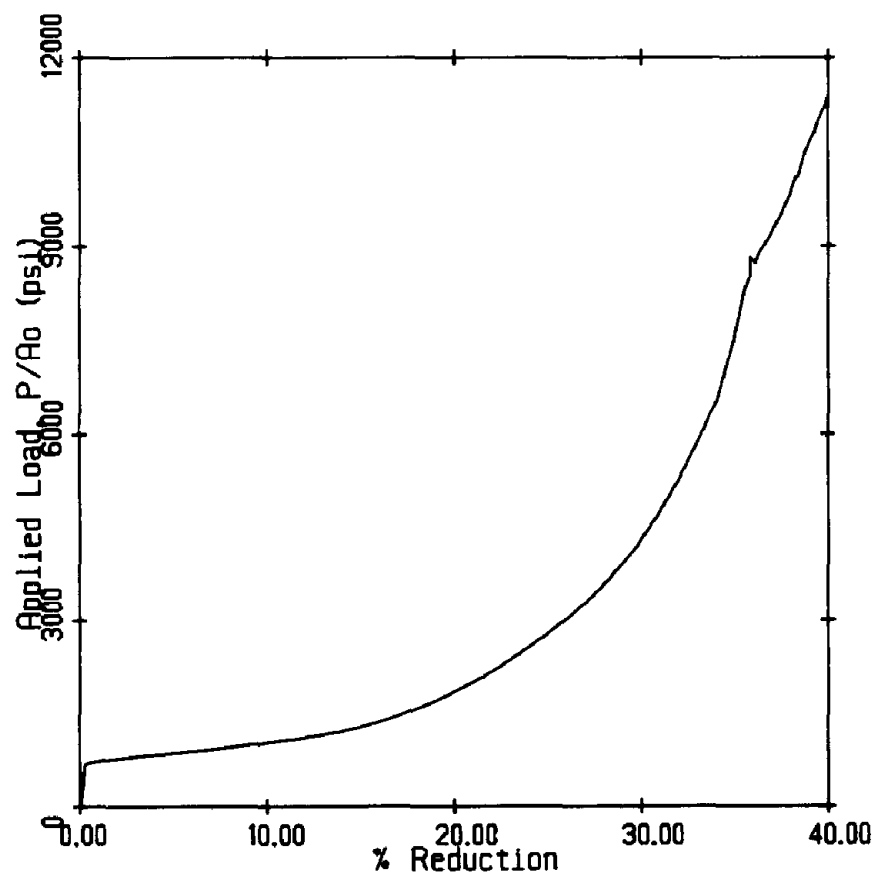

Fig. 23. Applied loads evaluated from element stresses under the rigid punch during a head forming process. $A_{0}=12.75 \mathrm{in}^{2}$. 


\section{Conclusion}

A finite element analysis based on updated Lagrangian formulations has been carried out to solve large deformation elasto-plasticity problems involving contact with friction. Four examples have been analyzed to examine the quality of the solutions through the comparison with some published results.

The first problem was a uniaxial tension test which offered a critical test on the plasticity model and the consideration of finite strain coded in the computer program. The second problem was an upsetting process of a cylindrical block. Locking phenomena due to the overstiff response of the 4CST element under the assumption of sticking punch-billet interface were discussed. Some considerations to resolve this difficulty were given. The emphasis has been laid on the importance of the choices of the increment size and the finite element discretization. The third example, an axisymmetric indentation problem, was selected to check the proposed friction algorithms. Finally, a head forming process was presented to show a practical application of the formulations in this article.

As the natural course of development, an extension of the present work would be to take account of the temperature effects in both the material constants and the surface properties. Lacking of experimental data and the complicated coupled problems pose a challenging field for research.

\section{Acknowledgment}

The authors are grateful to Prof. J.T.Oden in the University of Texas at Austin for his invaluable comments on the literature survey and the friction law. Thanks are also due to CRIM (Center of Robotics and Integrated Manufacturing), and the University of Michigan for providing computer time for the present work.

\section{References}

[1] J.H. Argyris, Continua and discontinua, Proc. First Conf. Matrix Methods in Struct. Mech., AFFDL-TR-6680 (1966) 11-190.

[2] G.G. Pope, A discrete element method for the analysis of plane elasto-plastic stress problems, Aeron. Quart. 17 (1966) 83-104.

[3] P.V. Marcal and I.P. King, Elastic-plastic analysis of two-dimensional stress systems by the finite element method, Internat. J. Mech. Sci. 9 (1967) 143-155.

[4] Y. Yamada, N. Yoshimura and T. Sakurai, Plastic stress strain matrix and its application for the solution of elastic-plastic problems by the finite element method, Internat. J. Mech. Sci. 10 (1967) 343-354.

[5] Y. Yamada, T. Kawai, N. Yoshimura and T. Sakurai, Analysis of the elastic-plastic problems by the matrix displacement method, Proc. 2nd Conf. on Matrix Methods in Struct. Mech., AFFDL-TR-68-150 (1969) 1271-1299.

[6] O.C. Zienkiewicz, S. Valliappan and I.P. King, Elastoplastic solutions of engineering problems-'Initial stress', finite element approach, Internat. J. Numer. Meths. Engrg. (1969) 75-100.

[7] M.J. Turner, F.H. Dill, H.C. Martin and R.J. Melosh, Large deflections of structures subjected to heating and external loads, J. Aerospace Sci. 27 (1960) 97-102.

[8] R.H. Gallagher, R.A. Gellatly, J. Radlog and R.H. Mallett, A discrete element procedure for thin-shell instability analysis, AIAA J. 5 (1967) 138-145. 
[9] K.K. Kapur and J. Hartz, Stability of plates using the finite element method, J. Engrg. Mech. Div., Proc. ASCE 92 (1966) 177-196.

[10] H.C. Martin, On the derivation of stiffness matrices for the analysis of large deflection and stability problems, Proc. 1st Conf. on Matrix Methods in Struct. Mech., AFFDL-TR-66-80 (1966) 697-715.

[11] H.C. Martin, Finite elements and the analysis of geometrically nonlinear problems, in: R.H. Gallagher, Y. Yamada and J.T. Oden, eds., Recent Advances in Matrix Methods of Struct. Analysis and Design, Proc. U.S.-Japan Seminar on Matrix Methods in Struct. Analysis and Design (1969) 343-381.

[12] J.T. Oden, Numerical formulation of non-linear elasticity problem, J. Struct. Div., Proc. ASCE 93 (1967) 235-255.

[13] J.T. Oden, Finite plane strain of incompressible elastic solids by the finite element method, Aeron. Quart. 19 (1968) 254-264.

[14] J.T. Oden, Finite element analysis of nonlinear problems in the dynamical theory of coupled thermoclasticity, Nucl. Engrg. Design 10 (1969) 465-475.

[15] J.R. Besseling, Matrix analysis of creep and plasticity problems, Proc. First Conf. on Matrix Methods in Struct. Mech., AFFDL-TR-66-80 (1966) 655-678.

[16] J.W. Wissmann, Nonlinear structural analysis: Tensor formulation, Proc. 1st Conf. on Matrix Methods in Struct. Mech., AFFDL-TR-66-80 (1966) 679-696.

[17] R.H. Mallett and P.V. Marcal, Finite element analysis of nonlinear structures, J. Struct. Div., Proc. ASCE (1968) 2081-2105.

[18] D.W. Murray and E.L. Wilson, Finite-element large deflection analysis of plates, J. Engrg. Mech. Div., Proc. ASCE 95 (1969) 143-165.

[19] P.V. Marcal, Large deflection analysis of elastic-plastic sheets of revolution, AIAA/ASME 10th Struct. Dynamics and Material Conf. (1969) 369-379.

[20] T. Kawai, Finite element analysis of the geometrically nonlinear problems of elastic plates, in: R.H. Gallagher, Y. Yamada and J.T. Oden, eds., Recent Advances in Matrix Methods of Struct. Analysis and Design (1970) 383-414.

[21] B.J. Hartz and N.D. Nathan, Finite element formulation of geometrically nonlinear problems of elasticity, in: R.H. Gallagher, Y. Yamada and J.T. Oden, eds., Recent Advances in Matrix Methods of Struct. Analysis and Design (1970) 415-417.

[22] J.T. Oden and J.E. Key, Numerical analysis of finite axisymmetric deformation of incompressible elastic solids of revolution, Internat. J. Solids Struct. 6 (1970) 497-518.

[23] J.T. Oden, Finite element applications in nonlinear structural analysis, Proc. Symp. Appl. Finite Element Methods Civil Engrg., Vanderbilt University, Nashville, TN. (1969) 419-456.

[24] J.T. Oden, Finite element formulation of problems of finite deformation and irreversible thermodynamics of nonlinear continua, in: R.H. Gallagher, Y. Yamada and J.T. Oden, eds., Recent Advances in Matrix Methods of Struct. Analysis and Design (1970) 383-414.

[25] J.T. Oden, Finite Elements of Nonlinear Continua (McGraw-Hill, New York, 1972).

[26] H.D. Hibbit, P.V. Marcal and J.R. Rice, A Finite element formulation for problems of large strain and large displacement, Internat. J. Solids Struct. (1970) 1069-1086.

[27] R.M. McMeeking and J.R. Rice, Finite-element formulation for problems of large elastic-plastic deformation, Internat. J. Solids Struct. 11 (1975) 601-616.

[28] R. Hill, Some basic principles in the mechanics of solids without a natural time, J. Mech. Phys. Solids 7 (1959) 209-225.

[29] A. Needleman, A numerical study of necking in circular cylindrical bars, J. Mech. Phys. Solids 20 (1972) 111-127.

[30] P.K. Larsen and E.P. Popov, A note on incremental equilibrium equations and approximate constitutive relations in large inelastic deformation, Acta Mech. 19 (1974) 1-14.

[31] S.W. Key, A finite element procedure for the large deformation dynamic response of axisymmetric solids, Comput. Meths. Appl. Mech. Engrg. 4 (1974) 195-218.

[32] J.R. Osias and J.L. Swedlow, Finite elasto-plastic deformation-I. Theory and numerical examples, Internat. J. Solids Struct. 10 (1974) 321-339.

[33] K.J. Bathe, E. Ramm and E.L. Wilson, Finite element formulations for large deformation dynamic analysis, Internat. J. Numer. Meths. Engrg. 9 (1975) 353-386. 
[34] M. Kleiber, Lagrangian and Eulerian finite element formulation for large strain elasto-plasticity, Bull. Acad. Polon. Sci. Ser. Sci. Tech. 23 (1975) 117-126.

[35] W. Wunderlich, Incremental formulations for geometrically nonlinear problems, in: K.J. Bathe, J.T. Oden and W. Wunderlich, eds., Formulations and Geometrical Algorithms in Finite Element Analysis: U.S.Germany Symposium (1976) 193-240.

[36] J.H. Argyris and M. Kleiber, Incremental formulation in nonlinear mechanics and large strain elastoplasticity - Natural approach. Part I, Comput. Meths. Appl. Mech. Engrg. 11 (1977) 215-247.

[37] J.H. Argyris, J. St. Doltsinis and M. Kleiber, Incremental formulation in nonlinear mechanics and large strain elasto-plasticity-Natural approach. Part II, Comput. Meths. Appl. Mech. Engrg. 14 (1978) 259-294.

[38] S. Cescotto, F. Frey and G. Fonder, Total and updated Lagrangian descriptions in nonlinear structural analysis: A unified approach, in: R. Glowinski, ed., Energy Methods in Finite Element Analysis (Wiley, New York, 1979) 203-296.

[39] S.N. Atluri, On rate principles for finite strain analysis of elastic and inelastic nonlinear solids, in: Recent Research on Mechanical Behavior of Solids (Univ. of Tokyo Press, Tokyo, 1979) 79-107.

[40] S.N. Atluri, On some new general and complementary energy theorems for the rate problems in finite strain, classical elastoplasticity, J. Struct. Mech. 8 (1980) 61-92.

[41] M.S. Gadala, G.A.E. Oravas and M.A. Dokainish, A consistent Eulerian formulation of large deformation problems in statics and dynamics, Internat. J. Non-Linear Mech. 18 (1983) 21-35.

[42] K. Mattiasson, On the co-rotational finite element formulation for large deformation problems, Ph.D. Dissertation, Chalmers University of Technology, Goteborg, Sweden, 1983.

[43] L.G. Chen, Necking in uniaxial tension, Internat. J. Mech. Sci. 25 (1983) 47-57.

[44] E.H. Lee, R.L. Mallett and W.H. Yang, Stress and deformation analysis of the metal extrusion process, Comput. Meths. Appl. Mech. Engrg. 10 (1977) 339-353.

[45] E.H. Lee, R.L. Mallett and R.M. McMeeking, Stress and deformation analysis of metal forming processes, in: R.F. Jones, Jr., H. Armen and J.T. Fong, eds., Numerical Modeling of Manuf. Processes, PVP-PB-025. ASME (1977) 19-33.

[46] Y. Yamada, A.S. Wifi and T. Hirakawa, Analysis of large deformation and stress in metal forming processes by the finite element method, in: H. Lippmann, ed., Metal Forming Plasticity, IUTAM Symposium Tutzing/Germany (1978) 158-176.

[47] J.C. Nagtegaal and J.E. De Jong, Some computational aspects of elastic-plastic large strain analysis, in: J.T. Oden, ed., Computational Methods in Nonlinear Mechanics (North-Holland, Amsterdam, 1980) 303-339.

[48] C.R. Boer, P. Gudmundson and N. Rebelo, Comparison of elastoplastic FEM and experiments for cylinder upsetting, in: J.F.T. Pittman, et al., eds., Numerical Methods in Industrial Forming Processes (Pineridge Press, Swansea, U.K., 1982) 217-226.

[49] H. Kudo and S. Matsubara, Joint examination project of validity of various numerical methods for the analysis of metal forming processes, in: H. Lippmann, ed., Metal Forming Plasticity, IUTAM Symposium Tutzing/Germany (1978) 378-403.

[50] M. Gotoh, A finite element analysis of general deformation of sheet metals, Internat. J. Numer. Meths. Engrg. 8 (1974) 731-741.

[51] N.M. Wang and B. Budiansky, Analysis of sheet metal stamping by a finite-element method, J. Appl. Mech. 45 (1978) 73-82.

[52] S.I. Oh and S. Kobayashi, Finite clement analysis of plane-strain sheet bending, Internat. J. Mech. Sci. 22 (1980) 583-594.

[53] A.S. Wifi, An incremental complete solution of the stretch-forming and deep-drawing of a circular blank using a hemispherical punch, Internat. J. Mech. Sci. 18 (1976) 23-31.

[54] A.S. Wifi, Finite element correction matrices in metal forming analysis (with application to hydrostatic bulging of a circular sheet), Internat. J. Mech. Sci. 24 (1982) $393-406$.

[55] S.W. Key, R.D. Krieg and K.J. Bathe, On the application of the finite element method to metal forming processes-Part I, Comput. Meths. Appl. Mech. Engrg. 17/18 (1979) 597-608.

[56] J.H. Argyris and J. St. Doltsinis, On the large strain inelastic analysis in natural formulation, Part I: Quasi-static problems, Comput. Meths. Appl. Mech. Engrg. 20 (1979) 213-251.

[57] J.H. Argyris and J. St. Doltsinis, On the large strain inelastic analysis in natural formulation, Part II: Dynamic problems, Comput. Meths. Appl. Mech. Engrg. 21 (1980) 91-126. 
[58] J.H. Argyris and J. St. Doltsinis, On the natural formulation and analysis of large deformation coupled thermomechanical problems, Comput. Meths. Appl. Mech. Engrg. 25 (1981) 195-253.

[59] J.H. Argyris, J. St. Doltsinis, W.C. Knudson, J. Szimmat, K.J. William and H. Wustenberg, Eulerian and Lagrangian techniques for elastic and inelastic large deformation processes, in: J.T. Oden, ed., Computational Meths. in Nonlinear Mechanics (North-Holland, Amsterdam, 1980) 13-66.

[60] L.M. Taylor, A finite element analysis for large deformation metal forming problems involving contact and friction, Ph.D. Dissertation, University of Texas at Austin, 1981.

[61] N. Kikuchi and J.H. Cheng, Finite element analysis of large deformation problems including unilaterial contact and friction, in: S.N. Atluri, ed., Computer Methods for Nonlinear Solids and Struct. Mechanics, AMD Vol. 54 (ASME, New York, 1983) 121-132.

[62] S. Yaghmai and E.P. Popov, Incremental analysis of large deflections of shells of revolution, Internat. J. Solids Struct. 7 (1971) 1375-1393.

[63] G.A. Dupuis, H.D. Hibbitt, S.F. McNamara and P.V. Marcal, Nonlinear material and geometric behavior of shell structures, Comput. \& Struct. 1 (1971) 223-239.

[64] J.H. Heifitz and C.J. Constantino, Dynamic response of nonlinear media at large strains, J. Engrg. Mech. Div., Proc. ASCE 98 (1972) 1511-1527.

[65] K.J. Bathe and H. Ozdemir, Elastic-plastic large deformation static and dynamic analysis, Comput. \& Struct. 6 (1976) 81-92.

[66] K.J. Bathe and S. Bolourchi, Large displacement analysis of three-dimensional beam structures, Internat. J. Numer. Meths. Engrg. 14 (1979) 961-986.

[67] J.H. Argyris, H. Balmer, M. Kleiber and U, Hindenlang, Natural description of large inelastic deformations for shells of arbitrary shape-Application of trump element, Comput. Meths. Appl. Mech. Engrg. 22 (1980) 361-389.

[68] C.H. Lee and S. Kobayashi, New solutions to rigid-plastic deformation problems using a matrix method, J. Engng. Ind., Trans. ASME 7 (1973) 865-873.

[69] S. Kobayashi, Rigid-plastic finite element analysis of axisymmetric metal forming processes, in: R.F. Jones, Jr. et al., eds., Numerical Modeling of Manuf. Process (ASME, New York, 1977) 49-65.

[70] C.C. Chen and S. Kobayashi, Rigid-plastic finite element analysis of ring compression, Appl. Numer. Methods to Forming Processes, AMD-28 (1978) 163.

[71] O.C. Zienkiewicz and I.C. Cormeau, Visco-plasticity and creep in elastic solids-A unified numerical solution approach, Internat. J. Numer. Meths. Engrg. 8 (1974) 821-845.

[72] O.C. Zienkiewicz and P.N. Godbole, Flow of plastic and visco-plastic solids with special reference to extrusion and forming processes, Internat. J. Numer. Meths. Engrg. 8 (1974) 3-16.

[73] O.C. Zienkiewicz and P.N. Godbole, A penalty function approach to problems of plastic flow of metals with large surface deformations, J. Strain Analysis 10 (1975) 180-183.

[74] O.C. Zienkiewicz, E. Onate and J.C. Heinrich, A general formulation for coupled thermal flow of metals using finite elements, Internat. J. Numer. Meths. Engrg. 17 (1981) 1497-1514.

[75] E. Onate and O.C. Zienkiewicz, A viscous shell formulation for the analysis of thin sheet metal forming, Internat. J. Mech. Sci. 25 (1983) 305-335.

[76] P.R. Dawson and E.G. Thompson, Finite element analysis of steady-state elasto-visco-plastic flow by the initial stress-rate method, Internat. J. Numer. Meths. Engrg. 12 (1978) 47-57.

[77] J.R. Rice and D.M. Tracey, Computational fracture mechanics, in: S.J. Fenves, ed., Proc. Symp. Numer. and Comput. Meths. in Struct. Mech. (Academic Press, New York, 1973) 585.

[78] R.D. Krieg and D.B. Krieg, Accuracies of numerical solution methods for the elastic-perfectly plastic model, J. Press. Vess. Jechn., Trans. ASME 99 (1977) 510-515.

[79] H.L. Schreyer, R.F. Kulak and J.M. Kramer, Accurate numerical solutions for elastic-plastic models, J. Press. Vess. Techn., Trans. ASME 101 (1979) 226-234.

[80] M.A. Crisfield, A fast incremental iterative solution procedure that handles "snap-through', Comput. \& Struct. 13 (1981) 55-62.

[81] D.M. Tracey and C.E. Freese, Adaptive load incrementation in elastic-plastic finite element analysis, Comput. \& Struct. 13 (1981) 45-53.

[82] C. Nyssen. An effective and accurate iterative method, allowing large incremental steps, to solve elasticplastic problems, Comput, \& Struct. 13 (1981) 63-71. 
[83] L.R. Hermann, Elasticity equations for incompressible and nearly incompressible materials by a variational theorem, AIAA J. 3 (1965) 1896-1900.

[84] J.C. Nagtegaal, D.M. Parks and J.R. Rice, On numerically accurate finite element solutions in the fully plastic range, Comput. Meths. Appl. Mech. Engrg. 4 (1974) 153-177.

[85] O.C. Zienkiewicz, R.L. Taylor and J.M. Too, Reduced integration technique in general analysis of plates and shells, Internat. J. Numer. Meths. Engrg. 3 (1971) 275-290.

[86] S.F. Pawsey and R.W. Clough, Improved numerical integration of thick shell finite elements, Internat. J. Numer. Meths. Engrg. 3 (1971) 575-586.

[87] D.J. Naylor, Stresses in nearly incompressible materials by finite elements with application to the calculation of excess pore pressures, Internat. J. Numer. Meths. Engrg. 8 (1974) 443-460.

[88] K.J. Bathe, M.D. Synder, A.P. Cimento and R.W. Donald, On some current procedures and difficulties in finite element analysis of elastic-plastic response, Comput. \& Structures 12 (1980) 607-624.

[89] J.C. Nagtegaal and F.E. Veldpaus, Analysis of metal forming problems with an improved finite strain plasticity formulation, in: J.F.T. Pittman et al., eds., Numerical Meths. in Industrial Forming Processes (Pineridge Press, Swansea, U.K. 1982) 1-15.

[90] A. Signorini, Questioni di elastostatica linearizzata e semilinearizzata, Rend. di Matem. e delle Sur. Appl. 18 (1959).

[91] G. Fichera, Un teorema generale de semicontinuita per gli integrali multipli e sue applicazioni alla fiscamatematica, Atti del Convegno Lagrangiano, Torino (1963) 138-151.

[92] J.J. Kalker, Aspects of contact mechanics, in: A.D. de Peter and J.J. Kalkar, eds., The Mechanics of the Contact between Deformable Bodies, Proc. Symp. IUTAM (Delft Univ. Press, Delft, 1975) 1-25.

[93] B. Fredriksson, G. Rydholm and P. Sjoblom, Variational inequalities in structural mechanics with emphasis on contact problems, Finite Elements in Nonlinear Mechanics, Int. Conf. on Finite Elements in Nonlinear Solid and Structural Mechanics (1977) 863-883.

[94] I. Hlavacek and J. Lovisek, A finite-element analysis for the signorini problem in plane elastostatics, Aplikace Matematiky 22 (1922) 215-218.

[95] N. Kikuchi and J.T. Oden, Contact Problems in Elasticity (SIAM, Philadelphia, PA, 1981).

[96] N. Kikuchi and Y.J. Song, Penalty/finite-element approximations of a class of unilateral problems in linear elasticity, Quart. Appl. Math. XXXIX (1981) 1-22.

[97] N. Kikuchi and K. Skalski, An elasto-plastic rigid punch problem using variational inequalities, Arch. Mech. 33 (1981) 865-877.

[98] J.T. Oden and N. Kikuchi, Finite element methods for constrained problems in elasticity, Internat. J. Numer. Meths. Engrg. 18 (1982) 701-725.

[99] S.K. Chan and I.S. Tuba, A finite element method for contact problems of solid bodies-Part I. Theory and validation, Part II. Application to turbine blade fastenings, Internat. J. Mech. Sci. 13 (1971) 615-639.

[100] T. Tsuta and S. Yamaji, Finite-element analysis of contact problem, Theory and Practice in Finite-Element Struct. Anal. (Tokyo Univ. Press, Tokyo, 1973) 177-194.

[101] S. Ohte, Finite element analysis of elastic contact problems, Bull. JSME 16 (1973) 797-804.

[102] A. Francavilla and O.C. Zienkiewicz, A note on numerical computation of elastic contact problems, Internat. J. Numer. Meths. Engrg. 9 (1975) 913-924.

[103] M. Okabe and N. Kikuchi, An application of penalty methods to a two-body contact problem, Proc. 3rd EMD Speciality Conf., ASCE (1979).

[104] T.D. Sachdeva and C.V. Ramakrishnan, A finite element solution for the two-dimensional elastic contact problems with friction, Internat. J. Numer. Meths. Engrg. 17 (1981) 1257-1271.

[105] I. Zeid and J. Padovan, Finite element modeling of rolling contact, Comput. \& Struct. 14 (1981) 163-170.

[106] M. Mazurkiewicz and W. Ostachowicz, Theory of finite element method for elastic contact problems of solid bodies, Comput. \& Struct. 17 (1983) 51-59.

[107] F.M. Guerra and R.V. Browning, Comparison of two slideline methods using ADINA, Comput. \& Struct. 17 (1983) 819-834.

[108] H. Schäfer, A contribution to the solution of contact problems with the aid of bond elements, Comput. Meths. Appl. Mech. Engrg. 6 (1975) 335-354.

[109] Y. Yamada, Y. Ezawa, I. Nishiguchi and M. Okabe, Handy incorporation of hond and singular elements in finite element solution routine, Trans. 5th Internat. Conf. on SMIRT (1979). 
[110] T.J.R. Hughes, R.L. Taylor, L.J.L. Sackman, A. Curnier and W. Kanoknukulchai, A finite element method for a class of contact-impact problems, Comput. Meths. Appl. Mech. Engrg. 8 (1982) 1505-1520.

[111] D. Talaslidis and P.D. Panagiotopoulos. A linear finite element approach to the solution of the variational inequalities arising in contact problems on structural dynamics, Internat. J. Numer. Meths. Engrg. 18 (1982) $1505-1520$.

[112] D. Osmont, Computation of the dynamic response of structures with unilateral constraints (contact)Comparison with experimental results, Comput. Meths. Appl. Mech. Engrg. 34 (1982) 847-859.

[113] Y. Seguchi, A. Shindo, Y. Tomita and M. Sunohara, Sliding rule of friction in plastic forming of metal, Comput. Methods in Nonlinear Mechanics (University of Texas at Austin, Austin, TX, 1974) 683-692.

[114] B. Fredriksson, Finite element solution of surface nonlinearities in structural mechanics with special emphasis to contact and fracture mechanics problems, Comput. \& Structures 6 (1976) 281-290.

[115] J. Petersson, Application of the finite element method in the analysis of contact problems, Finite Elements in Nonlinear Mechanics, Internat. Conf. on Finite Elements in Nonlinear Solid and Struct. Mechanics (1977) $845-862$.

[116] R. Michalowski and Z. Mroz, Associated and non-associated sliding rules in contact friction problems, Arch. Mech. 30 (1978) 259-276.

[117] N. Okamoto and M. Nakazawa, Finite element incremental contact analysis with various frictional conditions, Internat. J. Numer. Meths. Engrg. 14 (1979) 337-357.

[118] H. Hencky, Zur Theorie plastischer Deformationen und der hierdurch im Material hervorgerufenen Nebenspannungen, Z. Angew. Math. Mech. (1924) 312-316.

[119] B. Budiansky, A reassessment of deformation theories of plasticity, J. Appl. Mech. 26 (1959) 259-264.

[120] J. Christoffersen and J.W. Hutchinson, A class of phenomenological corner theories of plasticity, J. Mech. Phys. Solids 27 (1979) 465-487.

[121] L.B. Freund, Constitutive equations for elastic-plastic materials at finite strain, Internat. J. Solids Struct. 6 (1970) 1193-1209.

[122] M. Tanaka, Large deflection analysis of elastic-plastic circular plates with combined isotropic and kinematic hardening, Ingenieur-Archiv 41 (1972) 342-356.

[123] H.T. Hahn, A finite-deformation theory of plasticity, Internat. J. Solids. Struct. 10 (1974) 111-121.

[124] S.W. Key, J.H. Biffle and R.D. Krieg, A study of the computational and theoretical differences to two finite strain elastic-plastic constitutive models, in: K.J. Bathe, J.T. Oden and W. Wunderlich, eds., Formulations and Geometrical Algorithms in Finite Element Analysis: U.S.-German Symposium (1976) 321-352.

[125] E. Shiratori, K. Ikegami and F. Yoshida, Analysis of stress-strain relations by using an anisotropic hardening plastic potential, J. Mech. Phys. Solids 27 (1979) 213-229.

[126] K. Axelsson and A. Samuelsson, Finite element analysis of elastic-plastic materials displaying mixed hardening, Internat. J. Numer. Meths. Engrg. 14 (1979) 211-225.

[127] P.A. Dashner, A finite strain work-hardening theory for rate independent elasto-plasticity, Internat. J. Solids Struct. 15 (1979) 519-528.

[128] K. Hashiguchi, Constitutive equations of elastoplastic materials with anisotropic hardening and elastic-plastic transition, J. Appl. Mech. 48 (1981) 297-301.

[129] J. Mandel, Sur la définition de la vitesse de déformation elastique et sa relation avec la vitesse de contrainte, Inter. J. Solids Struct. 17 (1981) 871-878.

[130] S. Nemat-Nasser, On finite deformation elasto-plasticity, Internat. J. Solids Struct. 18 (1982) 857-872.

[131] F. Sidoroff, Incremental constitutive equation for large strain elasto-plasticity, Internat. J. Engrg. Sci. 20 (1982) $19-26$.

[132] T. Tukuora, Plastic materials with continuous transition between loading and unloading states, Arch. Mech. 34 (1982) 187-197.

[133] S.W. Key and R.D. Krieg, On the numerical implementation of inelastic time dependent and time independent, finite strain constitutive equations in structural mechanics, Comput. Meths. Appl. Mech. Engrg. 33 (1982) $439-452$.

[134] E.H. Lee, R.L. Mallett and T.B. Weitheimer, Stress analysis for anisotropic hardening in finite-deformation plasticity, J. Appl. Mech. 50 (1983) 554-560.

[135] L. Palgen and D.C. Drucker, The structure of stress-strain relations in finite elasto-plasticity, Internat. J. Solids Struct. 19 (1983) 519-531. 
[136] S.N. Atluri, On constitutive relations at finite strain: Hypo-elasticity and elasto-plasticity with isotropic or kinematic hardening, Report No. GIT-CAM-SNA-83-16, Georgia Institute of Technology, 1983.

[137] S. Nemat-Nasser, On finite plastic flow of crystalline solids and geomaterials, J. Appl. Mech. 50 (1983) $1114-1126$.

[138] R. Hill, The Mathematical Theory of Plasticity (Oxford Univ. Press, Oxford, 1950).

[139] J.G. Oldroyd, On the formulation of rheological equations of state, Proc. Roy. Soc. A 220 (1950) 523-541.

[140] B.A. Cotter and R.S. Rivlin, Tensors associated with time-dependent stress, Quart. Appl. Math. 13 (1955) $177-182$.

[141] C. Truesdell, The simplest rate theory of pure elasticity, Comm. Pure Appl. Math. 8 (1955) 123-132.

[142] W. Prager, An elementary discussion of definitions of stress rate, Quart. Appl. Math. 18 (1961) 403-407.

[143] G.C. Johnson and D.J. Bammann, A discussion of stress rates in finite deformation problems, Sandia Report, SAND82-8821, 1982.

[144] E.H. Lee and T.B. Wertheimer, Deformation analysis of simple shear loading with anisotropic hardening in finite-deformation plasticity, in: S.N. Atluri, ed., Computer Meths. Nonlinear Solids and Struct. Mech., AMD Vol. 54 (ASME, New York, 1983) 145-154.

[145] Y.F. Dafalias, Corotational rates for kinematic hardening at large plastic deformations, J. Appl. Mech. 50 (1983) 561-565.

[146] A.J.M. Spencer, Continuum Mechanics (Longman, London, 1980).

[147] S. Nemat-Nasser, Decomposition of strain measures and their rates in finite deformation elastoplasticity, Internat. J. Solids Struct. 15 (1979) 155-166.

[148] E.H. Lee, Elastic-plastic deformation at finite strains, J. Appl. Mech. 36 (1969) 1-6.

[149] E.H. Lee, Some comments on elastic-plastic analysis, Internat. J. Solids Struct. 17 (1981) 859-872.

[150] A.E. Green and P.M. Naghdi, Some remarks on elastic-plastic deformation at finite strain, Internat. J. Engrg. Sci. 9 (1971) 1219-1229.

[151] J. Casey and P.M. Naghdi, A remark on the use of the decomposition $F=F_{\mathrm{e}} F_{\mathrm{p}}$ in plasticity, J. Appl. Mech. 47 (1980) 672-675.

[152] E.H. Lee and R.M. McMeeking, Concerning elastic and plastic components of deformation, Internat. J. Solids Struct. 16 (1980) 715-721.

[153] Y.C. Fung, Foundations of Solid Mechanics (Prentice-Hall, Englewood Cliffs, NJ, 1965).

[154] L.E. Malvern, Introduction to the Mechanics of a Continuous Medium (Prentice-Hall, Englewood Cliffs, NJ, 1969).

[155] Y. Tomita, Displacement constraints in large strain elastic-plastic finite element analysis, J. Japan Soc. Techn. Plasticity 22 (1981) 410-418, in Japanese.

[156] R. Courant, K. Friedrichs and H. Lewy, On the partial difference equations of mathematical physics, IBM J. 11 (1967) 215-234.

[157] W.I. Zangwill, Nonlinear programming via penalty functions, Management Science 13 (1967) 344-358.

[158] R. Courant, Variational methods for the solutions of problems of equilibrium and vibrations, Bull. Amer. Math. Soc. 49 (1943) 1-23.

[159] F.P. Bowden and D. Tabor, The Friction and Lubrication of Solids, Part II (Clarendon Press, London, 1964).

[160] J.S. Courtney-Pratt and E. Eisner, The effect of a tangential force on the contact of metallic bodies, Proc. Roy. Soc. A 238 (1957) 529-550.

[161] J.T. Oden and E.B. Pires, Nonlocal and nonlinear friction laws and variational principles for contact problems in elasticity, J. Appl. Mech. 50 (1983) 67-76.

[162] J.T. Oden and E.B. Pires, Numerical analysis of certain contact problems in elasticity with non-classical friction laws, Comput. \& Struct. 16 (1983) 481-485.

[163] O.C. Zienkiewicz, The Finite Element Method (McGraw-Hill, New York, 3rd ed., 1977).

[164] W.H. Chen, Necking of a bar, Internat. J. Solids Struct. 7 (1971) 685-717.

[165] D.M. Norris, B. Moran, J.K. Scudder and D.F. Quinones, A computer simulation of the tension test, J. Mech. Phys. Solids 26 (1978) 1-19.

[166] M. Saje, Necking of a cylindrical bar in tension, Internat. J. Solids Struct. 15 (1979) 731-742.

[167] N. Kikuchi, Remarks on 4CST-elements for incompressible materials, Comput. Meths. Appl. Mech. Engrg. 37 (1983) 109-123. 
[168] C.H. Lee and S. Kobayashi, Elastoplastic analysis of plane-strain and axisymmetric flat punch indentation by the finite-element method, Internat. J. Mech. Sci. 12 (1970) 349-370.

[169] C. Hardy, C.N. Baronet and G.V. Tordion, The elasto-plastic indentation of a half-space by a rigid sphere, Internat. J. Numer. Meths. Engrg. 3 (1971) 451-462.

[170] G. Dumas and C.N. Baronet, Elastoplastic indentation of a half-space by an infinitely long rigid circular cylinder, Internat. J. Mech. Sci. 13 (1971) 519-530.

[171] C.H. Lee, S. Masaki and S. Kobayashi, Analysis of ball indentation, Internat. J. Mech. Sci. 14 (1972) $417-426$.

[172] N. Kikuchi, Penalty/finite element approximations of a class of unilateral contact problems, in: J.N. Reddy, ed., Penalty Methods in Finite Element Methods (ASME, New York, 1982). 